\title{
Temporal inhibition in character identification
}

\author{
THOMAS A. BUSEY \\ Indiana University, Bloomington, Indiana
}

\begin{abstract}
Models of information processing tasks such as character identification often do not consider the nature of the initial sensory representation from which task-relevant information is extracted. An important component of this representation is temporal inhibition, in which the response to a stimulus may inhibit, or in some cases facilitate, processing of subsequent stimuli. Three experiments demonstrate the existence of temporal inhibitory processes in information processing tasks such as character identification and digit recall. An existing information processing model is extended to account for these effects, based in part on models from the detection literature. These experiments also discriminate between candidate neural mechanisms of the temporal inhibition. Implications for the transient deficit theory of dyslexia are discussed.
\end{abstract}

Despite the fact that we see the world as a continuous stream of visual stimulation, the input to the eyes consists of discrete quantal events. No two photons arrive at exactly the same time, and a visual system that reported the locations of arriving photons with infinite temporal fidelity would simply report a flurry of pinpoints, each representing one photon capture. In order to provide a coherent representation of a scene, the visual system must integrate information over some interval to temporally group photons coming from the same object. To quantify the notion of an integration interval, the response to a stimulus may be characterized as a temporal waveform that represents the result of temporally grouping the arriving photons to form some response that varies over time. Upon stimulus onset, this response may rise to some level and then decay sometime after stimulus offset. This response is termed the initial sensory response and is assumed to represent the output of neural mechanisms located early on in the visual stream (e.g., the retina and the primary visual cortex). It has long been known that the response to a stimulus outlasts its offset (see Coltheart, 1980, for an excellent review), and the characteristics of this initial sensory response reflect this fact. All subsequent visual processing is based on this initial representation, and thus the first step in characterizing the mechanisms that underlie object or character identification must address the nature of this initial sensory representation.

The nature of this sensory response function has previously been shown to be important for models of detection (Sperling \& Sondhi, 1968; Watson, 1986), intensityduration tradeoffs in information processing tasks (Loftus \& Ruthruff, 1994), metacontrast masking (Breitmeyer,

This research was funded in part by an NIMH predoctoral grant to T.A.B. and NINH Grant MH41637 to Geoffrey R. Loftus. Portions of this research were in partial fulfillment of a doctoral dissertation at the University of Washington. Correspondence should be addressed to T. A. Busey, Department of Psychology, Indiana University, Bloomington, IN 47405 (e-mail: busey@indiana.edu).
1984), completeness ratings and temporal integration performance (Loftus \& Irwin, 1998), synchrony judgment tasks (Hogben \& Di Lollo, 1974; Matin \& Bowen, 1976), and, more recently, character identification tasks (Busey \& Loftus, 1994; Loftus, Busey, \& Senders, 1993). In general, the shape of the sensory response function determines the visibility of the stimulus and the duration of its persistence, which in turn affects the amount of information that can be extracted from the stimulus and the amount of interaction with subsequent stimuli such as backward masks. Models of information processing tasks typically do not consider the nature of this initial sensory representation and instead focus on the processes that are involved with information extraction (e.g., Fisher, 1982; Rumelhart, 1970; Townsend, 1981). More recent models have begun to account for the continuous accumulation of information in information processing tasks (e.g., Eriksen \& Schultz, 1979; Gegenfurtner \& Sperling, 1993).

Characterizing the nature of the initial sensory representation and describing how its properties can affect performance in information processing tasks such as character recognition is the goal of the present article. To accomplish this goal, I consider models that have been applied to simple detection tasks and conjoin these with an existing information processing model. The resulting theory bridges the detection and information processing literatures and is used to address whether results derived from the detection of disks and gratings apply to the identification of letters as well.

Many researchers attempting to characterize the nature of the sensory response function have used a task known as the two-pulse paradigm to measure the temporal properties of the initial sensory representation. In the twopulse paradigm, a stimulus is presented twice for two brief pulses, each time in the same location, separated by an interstimulus interval (ISI) that typically ranges from 0 to $150 \mathrm{msec}$. Each pulse will produce a response in the visual system, and the two responses will interact in different ways for different ISIs. The time between pulses is varied to find the longest interval that still provides some 
evidence of interaction between the percepts of the two pulses. From this estimate of the integration interval, mathematical models are proposed that derive the shape of the sensory response function. This paradigm has been used with disks (e.g., Blackwell, 1963; Bouman \& van den Brink, 1952; Purcell \& Stewart, 1971), gratings (Watson \& Nachmias, 1977), Gaussian patches (Bergen \& Wilson, 1985), two-half displays (Hogben \& Di Lollo, 1974), and digits (Busey \& Loftus, 1994) in both detection and identification tasks.

As researchers derived estimates of the integration interval, several noted that the form of the interaction between the sensations elicited by the two pulses was more complex than simple summation. Ikeda (1965) provided surprising evidence for an inhibitory interaction at some delay intervals: When two 12.5 -msec pulses were separated by a 40 - to $70-\mathrm{msec}$ ISI, performance was worse than what would be expected based on probability summation of the individual responses. It appears from these data that the response to the first pulse interfered with or inhibited the processing of the second pulse. These effects have become known as temporal inhibition. Ikeda's (1965) innovation was to include a condition in which the second pulse could be of opposite contrast, which provides evidence that the two responses could facilitate each other at moderate ISIs (30-50 msec) despite the fact that one was light gray and the other was dark gray on a gray background. This facilitation between opposite-contrast pulses was described by Ikeda as additional evidence for temporal inhibition, under the assumption that a lightgray stimulus produces a response that is initially excitatory and then inhibitory. This inhibitory portion of the response can facilitate the excitatory portion of a response created by a dark-gray pulse, which is assumed to be compatible with the inhibitory portion of the first pulse's response.

Why might the visual system inhibit its own signal? Some clues come from the spatial domain: Lateral inhibition sharpens the percept of an edge in such a way that the response from receptors responding to the dark side of an edge is reduced as a result of inhibition coming from receptors responding to the light side of an edge. We might expect to find similar mechanisms in the temporal domain since the visual system attempts to sharpen a temporal edge caused by the appearance or disappearance of an object. The importance of inhibition has long been known in the masking literature, where lateral inhibition and several different mechanisms of temporal inhibition have accounted for metacontrast masking, backward masking, and saccadic suppression (see Breitmeyer, 1984).

The previous work on the effects of temporal inhibition has used simple stimuli in detection tasks. Using disks and spatial frequency gratings of various sizes, researchers found evidence for temporal inhibition in a wide range of conditions. However, none used more complex tasks and stimuli that are commonly associated with information processing tasks. For instance, grating detection requires only distinguishing the presence of some pattern relative to a blank screen, whereas character identification requires the classification of a pattern into one of many welllearned categories. In addition, letters are spatially compact and contain a broad range of spatial frequencies, whereas spatial frequency gratings are the opposite. Thus letters are expected to be mediated by multiple visual channels, whereas gratings might be mediated by only a few. Alexander, Xie, and Derlacki (1994) pointed out that simple stimuli such as gratings may not be sensitive to visual pathologies in persons with disorders of the visual system. Periodic test stimuli such as gratings may not reveal the extent of spatial undersampling in uncorrected refractive error, while the nonperiodic nature of letters may provide a better probe of this disorder. Thus the relatively complex spatial properties of letters suggest that findings from the detection literature may not apply directly to character identification.

There are several reasons why temporal inhibition is important for information processing tasks such as character identification. First, previous models have demonstrated that the nature of the initial sensory representation can affect the rate at which information is extracted from this representation (e.g., Busey \& Loftus, 1994). Temporal inhibition qualitatively changes the nature of the initial sensory representation and can therefore affect later processing stages as well. Second, temporal inhibition by definition affects the processing of stimuli that follow the offset of an object. Tasks such as backward masking, metacontrast masking, temporal integration, synchrony judgment, partial report, and rapid serial visual processing (RSVP) may all be influenced to some degree by temporal inhibition. Third, temporal inhibition may provide a signature of the underlying pathways or mechanisms at work in a given task. By delimiting the range of stimuli and tasks that show evidence of temporal inhibition, we can draw conclusions about the properties of the mechanisms that produce this effect. Finally, evidence for temporal inhibition in information processing tasks such as character identification may have implications for disabilities such as dyslexia, in which a possible deficit in processing high temporal frequencies has been suggested (Lovegrove, Garzia, \& Nicholson, 1990; Williams \& Lecluyse, 1990). I return to this final point in the General Discussion.

It is not at all clear a priori that information processing tasks such as character identification will produce the temporal inhibition effects seen with disks and gratings. In the spatial domain, Solomon and Pelli (1994) demonstrated that letters and gratings were mediated by the same spatial frequency channels. An important mediator of temporal inhibition is the spatial frequencies that are contained in the stimulus. Ohtani and Ejima (1988) demonstrated that temporal inhibition will only occur for sine-wave gratings with spatial frequencies lower than $.75-1.5$ cycles per degree, depending on the background luminance. Letter recognition may not depend on these low spatial frequencies. Solomon and Pelli demonstrated 
that letters are recognized primarily on the basis of spatial frequencies in an octave range centered at 3.2 cycles per degree ( 3 cycles per letter), which has a lower cutoff around 1.5 cycles per degree. If the spatial frequencies that underlie letter identification are higher than those that produce temporal inhibition, we will not observe effects consistent with temporal inhibition in a character identification task.

Alexander et al. (1994) addressed whether the temporal characteristics of letters and gratings differ. They measured the spatial contrast sensitivity functions for Sloan letters at different sizes and D6 gratings at various spatial frequencies, and found systematic differences that depend on temporal frequency. The size of the Sloan letters is expressed as the minimum angle of resolution (MAR) in units of arc minutes, while a D6 grating is the sixth spatial derivative of a Gaussian function in units of cycles per degree. These stimuli were flickered at either 2 or $16 \mathrm{~Hz}$. At the $2-\mathrm{Hz}$ flicker rate, systematic differences existed between the two spatial contrast sensitivity functions, demonstrating that temporal frequency had a systematic effect on the contrast sensitivity function for the two patterns. Given this finding, we might expect differences in the temporal domain between experiments that used gratings or disks and the present experiments, which used digits. The letter stimuli selectively improve sensitivity relative to the gratings at the lowest temporal frequencies, suggesting that the letters might rely on lower temporal frequencies than gratings. As a result, letters may not show evidence for temporal inhibition since temporal inhibition helps the system preserve and respond to higher temporal frequencies.

The results of these studies do not provide a clear answer to the question of whether the mechanisms that produce temporal inhibition contribute to character identification tasks. The present article addresses this question by applying an existing information processing model to tasks adapted from the detection literature. The article is organized as follows. I begin with a brief summary of the experimental variables that influence temporal inhibition in detection tasks. I then briefly describe an existing information processing model that can be used to model the empirical results. In three different paradigms I provide empirical evidence that temporal inhibition does indeed exist in character identification tasks, and I show how an existing model can account for these effects. Finally, I discuss possible mechanisms that underlie temporal inhibition and its implications for other information processing tasks.

\section{Variables Affecting Temporal Inhibition}

Spatial frequency, stimulus size, and background adaptation level all have been shown to affect temporal inhibition; the results of a variety of studies are summarized in Table 1. On the basis of a meta-analysis of these findings, Ikeda (1986) concluded that for disks, inhibitory phenomena appear only when the stimulus size becomes larger than about $13^{\prime}$ of arc. However, the temporal delay between the pulses at which inhibition is largest is not affected by the size of the stimulus beyond $13^{\prime}$ of arc. A parametric study by Ohtani and Ejima (1988) demonstrated that for sine-wave gratings, temporal inhibition occurs only for gratings with spatial frequencies larger than $1.5-0.75$ cycles per degree, depending on the background luminance.

Blackwell (1963) found evidence for temporal inhibition only for stimuli shown at a background luminance of $10 \mathrm{fLs}$. Roufs (1973) used $1^{\circ}$ stimuli on three different background levels and found an inhibitory effect for stimuli shown at background levels of 42 trolands (td) and 1,200 tds, but not at $1 \mathrm{td}$. Thus it appears that inhibition depends on the background level, although the critical background level is not fully defined. As the visual system becomes exposed to higher and higher light levels, the integration period grows shorter and shorter. This fits with the intuitive model that a visual system should integrate over longer periods in low light levels to gain sensitivity, but integrate over shorter intervals in higher light levels to gain responsiveness to changes in the visual scene.

A number of different mathematical models have been developed to account for the effects of temporal inhibition; these are summarized in Table 2. Most of the models follow Ikeda's (1965) proposal that the visual system acts as a temporal filter, which has the effect of temporally blurring the rapid changes created by the onset and offset of each pulse and causing the response to a stimulus to persist and interact with subsequent pulses. The behavior of the temporal filter is characterized by an impulse response function that defines the severity of the temporal blurring. The models then include some form of nonlinearity and a decision-making rule that allows prediction of contrast thresholds.

\section{Conjoining Temporal Inhibition with an Information Processing Model}

The models of Ikeda (1965), Rashbass (1970), Sperling and Sondhi (1968), and Watson and Nachmias (1977) provide a springboard for the modern linear filter models that have been proposed to account for many of the relationships between stimulus variables and temporal sensitivity, summarized in Table 1. Each model differs in terms of the form of the impulse response function and the nature of the response mechanisms, but each has been limited to modeling simple detection tasks. Additional model components are required to account for information processing tasks such as character identification. A model that includes all of these characteristics is developed below as an extension of an extant theory known as the linear systems theory (LST), proposed by Loftus et al. (1993) and Busey and Loftus (1994).

Figure 1 shows the components of the LST model that can be used to (1) account for the temporal summation that occurs in the two-pulse data, (2) determine the tem- 
Table 1 Summary of Stimulus Conditions and Results for Various Studies on Variables Affecting Temporal Inhibition

\begin{tabular}{|c|c|c|c|c|c|}
\hline Authors & Test Size & Adapting Level & Inhibition & $t$ & Conditions \\
\hline Ikeda (1965) & $30^{\prime}$ & $\begin{array}{l}61.2 \mathrm{td} \\
328 \mathrm{td}\end{array}$ & $\begin{array}{l}\text { yes } \\
\text { yes }\end{array}$ & $\begin{array}{l}70 \\
53\end{array}$ &,,,+++--+-- \\
\hline Rashbass (1970) & $17^{\circ}$ & $700 \mathrm{td}$ & yes & 66 &,,,+++--+-- \\
\hline $\begin{array}{l}\text { Purcell \& } \\
\quad \text { Stewart (1971) }\end{array}$ & $38^{\prime}$ & $25 \mathrm{FL}$ & yes & - & \\
\hline Theodor (1972) & $10^{\prime}$ & $25 \mathrm{FL}$ & yes & - & ++ \\
\hline Roufs (1973) & $1^{\circ}$ & $\begin{array}{l}1 \mathrm{td} \\
42 \mathrm{td} \\
1,200 \mathrm{td}\end{array}$ & $\begin{array}{l}\text { no } \\
\text { yes } \\
\text { yes }\end{array}$ & $\begin{array}{l}\overline{56} \\
32\end{array}$ & ++ \\
\hline $\begin{array}{l}\text { Breitmeyer \& } \\
\quad \text { Ganz (1977) }\end{array}$ & $\begin{array}{l}1.0 \mathrm{c} / \mathrm{deg} \\
10 \mathrm{c} / \mathrm{deg}\end{array}$ & $5.0 \mathrm{FL}$ & $\begin{array}{l}\text { yes } \\
\text { no }\end{array}$ & 80 & Gratings: ++ \\
\hline $\begin{array}{l}\text { Watson \& } \\
\text { Nachmias (1977) }\end{array}$ & $\begin{array}{l}2^{\circ} \times 1.5^{\circ} \\
1.8 \mathrm{c} / \mathrm{deg} \\
3.5 \mathrm{c} / \mathrm{deg} \\
7.0 \mathrm{c} / \mathrm{deg} \\
10 \mathrm{c} / \mathrm{deg}\end{array}$ & $15 \mathrm{~cd} / \mathrm{m}^{2}$ & $\begin{array}{l}\text { yes } \\
\text { yes } \\
\text { no } \\
\text { no }\end{array}$ & $\begin{array}{l}60 \\
60\end{array}$ & Gratings:,,,+++--+-- \\
\hline $\begin{array}{l}\text { Meijer, van der } \\
\text { Wildt \& van den } \\
\text { Brink (1978) }\end{array}$ & $\begin{array}{l}5.5^{\prime} \\
11^{\prime} \\
11^{\prime}-- \\
13.7^{\prime} \\
66^{\prime}-- \\
96^{\prime}\end{array}$ & $120 \mathrm{td}$ & $\begin{array}{l}\text { no } \\
\text { no } \\
\text { no } \\
\text { yes } \\
\text { yes } \\
\text { yes }\end{array}$ & $\begin{array}{l}- \\
- \\
70 \\
70 \\
70\end{array}$ & ++ and some -- \\
\hline $\begin{array}{l}\text { Roufs \& } \\
\quad \text { Blommaert (1981) }\end{array}$ & $0.8^{\prime}$ & $1,200 \mathrm{td}$ & $\begin{array}{l}\text { no } \\
\text { yes }\end{array}$ & $\overline{60}$ & \\
\hline $\begin{array}{l}\text { Musselwhite \& } \\
\text { Jeffreys (1983) }\end{array}$ & $\begin{array}{l}\text { grid of } \\
\text { dots } 16^{\prime}\end{array}$ & $\begin{array}{l}50 \mathrm{~cd} / \mathrm{m}^{2} \\
2.5 \mathrm{~cd} / \mathrm{m}^{2}\end{array}$ & $\begin{array}{l}\text { no } \\
\text { no }\end{array}$ & & Recorded VEPs,,,+++--+-- \\
\hline $\begin{array}{l}\text { Bergen \& } \\
\quad \text { Wilson (1985) }\end{array}$ & $\begin{array}{l}1^{\circ} \\
0.125^{\circ}\end{array}$ & $\begin{array}{l}17.2 \mathrm{~cd} / \mathrm{m}^{2} \\
\mathrm{~cd} / \mathrm{m}^{2}\end{array}$ & $\begin{array}{l}\text { yes } \\
\text { yes }\end{array}$ & $\begin{array}{l}50 \\
50\end{array}$ & Difference of Gaussian:,+++-+- \\
\hline $\begin{array}{l}\text { Uchikawa \& } \\
\quad \text { Ikeda }(1986)\end{array}$ & $\begin{array}{l}45^{\prime} \\
\text { chrom. } \\
\text { achrom. }\end{array}$ & $0 \mathrm{~cd} / \mathrm{m}^{2}$ & $\begin{array}{l}\text { no } \\
\text { no } \\
\text { yes }\end{array}$ & $\frac{-}{40}$ & $\begin{array}{l}++,+-,-+,-- \text { for chromatic } \\
\& \text { achromatic stimuli }\end{array}$ \\
\hline Georgeson (1987) & $\begin{array}{l}1.5 \mathrm{c} / \mathrm{deg} \\
3 \mathrm{c} / \mathrm{deg} \\
6 \mathrm{c} / \mathrm{deg} \\
12 \mathrm{c} / \mathrm{deg}\end{array}$ & $16 \mathrm{~cd} / \mathrm{m}^{2}$ & $\begin{array}{l}\text { no } \\
\text { no } \\
\text { no }\end{array}$ & & $\begin{array}{l}++,+-,-+,-- \text { for above- } \\
\text { threshold and near-threshold } \\
\text { stimuli; found TI at all gratings } \\
\text { for above-threshold task }\end{array}$ \\
\hline Bowen (1989) & $0.5 \mathrm{c} / \mathrm{deg}$ & $10 \mathrm{td}$ & yes & & ++ suprathreshold \\
\hline $\begin{array}{l}\text { Burr \& Morrone } \\
\text { (1993) }\end{array}$ & $\begin{array}{l}1 \mathrm{c} / \mathrm{deg} \\
\text { chrom. } \\
\text { achrom. }\end{array}$ & $16 \mathrm{~cd} / \mathrm{m}^{2}$ & $\begin{array}{l}\text { no } \\
\text { yes }\end{array}$ & 80 & $\begin{array}{l}++,+-,-+,-- \text { for isoluminant } \\
\& \text { achromatic stimuli }\end{array}$ \\
\hline
\end{tabular}

Note- $t$, temporal delay of inhibition (in milliseconds); + , positive-contrast pulse; - , negative-contrast pulse. VEP, visual-evoked potential. This table is patterned after that of Ikeda (1986).

poral characteristics of the sensory representation used in a given visual task, and (3) provide evidence for temporal inhibition in information processing tasks. The onset and offset of the digits are represented as changes in contrast over time, defined as a function $f(t)$, which is then temporally low-pass filtered by the model into a sensory representation, $a(t)$, that is a temporally blurred representation of the physical stimulus. Information is then extracted from the sensory response at a rate $r(t)$, which is used to make a performance prediction for a stimulus condition. Each of these components is described below.

Characterization of the physical stimulus. The function $f(t)$ represents the contrast of a pulse stimulus over time, as illustrated in the top panel of Figure 1. The negative contrast condition of the two-pulse paradigm sets the contrast to $-\Phi$. The initial sensory representation engendered by the physical stimulus is generated by convolving the physical stimulus representation with an impulse response function, $g(t)$ :

$$
a(t)=f(t) * g(t)=\int_{-\infty}^{\infty} f(t) g(t-x) d x
$$

The result, $a(t)$, called the sensory response function, is shown in the bottom panel of Figure 1. The exact form of $g(t)$ is described below, but the effect is that $a(t)$ is a temporal blurring or filtering of the physical stimulus $f(t)$. The shape of the impulse response function, $g(t)$, dictates the type of temporal blurring. The sensory response function component of the LST theory $[a(t)]$ is based on work from Andrew Watson (Watson, 1986), 
Table 2

Characteristics of Models of Two-Pulse Performance

\begin{tabular}{|c|c|c|c|c|c|}
\hline Author & Stimuli & Model Specifics & $\begin{array}{l}\text { Nature of } \\
\text { Impulse } \\
\text { Response } \\
\text { Function }\end{array}$ & $\begin{array}{l}\text { Predicts } \\
\text { Inhibition }\end{array}$ & Conditions \\
\hline Ikeda (1965) & $30^{\prime}$ disks & $\begin{array}{l}\text { Derived a summation index from } \\
\text { which the temporal response } \\
\text { functions for positive and negative } \\
\text { stimuli may be inferred }\end{array}$ & $\begin{array}{l}\text { Unknown, but } \\
\text { has inhibitory } \\
\text { lobe }\end{array}$ & yes &,,+++--+ \\
\hline Rashbass (1970) & $17^{\circ}$ disks & $\begin{array}{l}\text { Assumed a low-pass linear filter } \\
\text { that is squared and integrated; } \\
\text { predicts elliptical relationship for } \\
\text { contrast thresholds }\end{array}$ & Unspecified & yes &,,+++--+ \\
\hline $\begin{array}{l}\text { Sperling \& } \\
\text { Sondhi (1968) }\end{array}$ & Gratings & $\begin{array}{l}\text { Nature of the temporal } \\
\text { inhibition changes with } \\
\text { background luminance level }\end{array}$ & $\begin{array}{l}\text { Biphasic, varies } \\
\text { with background } \\
\text { luminance level }\end{array}$ & yes & \\
\hline $\begin{array}{l}\text { Watson \& } \\
\quad \text { Nachmias (1977) }\end{array}$ & Gratings & $\begin{array}{l}\text { Extended Rashbass model to include } \\
\text { probability summation in time }\end{array}$ & Biphasic & yes &,,,+++--+-- \\
\hline Roufs (1973) & $1^{\circ}$ disks & $\begin{array}{l}\text { Peak detection model conjoined } \\
\text { with low-pass linear filter }\end{array}$ & Biphasic & yes & ++ \\
\hline $\begin{array}{l}\text { Uchikawa \& } \\
\text { Ikeda (1986) }\end{array}$ & $\begin{array}{l}\text { Isoluminant } \\
\text { disks }\end{array}$ & $\begin{array}{l}\text { Adopted Watson's PST model to } \\
\text { predict isoluminant thresholds; } \\
\text { no inhibition found in } \\
\text { luminant stimuli }\end{array}$ & Monophasic & no & ++ \\
\hline $\begin{array}{l}\text { Roufs \& } \\
\quad \text { Blommaert (1981) }\end{array}$ & $\begin{array}{l}1^{\circ} \text { foveal } \\
\text { disk }\end{array}$ & $\begin{array}{l}\text { Derived form of impulse response } \\
\text { function by looking at interaction } \\
\text { of two responses at peak height; } \\
\text { criticized by Watson for ignoring } \\
\text { effects of probability summation } \\
\text { in time. }\end{array}$ & $\begin{array}{l}\text { Triphasic, with } \\
\text { an initial } \\
\text { inhibitory lobe }\end{array}$ & yes & ++ \\
\hline $\begin{array}{l}\text { den Brinker } \\
\quad(1989,1990)\end{array}$ & $1^{\circ}$ disk & $\begin{array}{l}\text { Adopted Roufs \& Blommaert } \\
\text { model, but accounted for addition } \\
\text { of temporal inhibition for higher } \\
\text { background levels }\end{array}$ & $\begin{array}{l}\text { Fourth-order } \\
\text { multiphasic } \\
\text { with an initial } \\
\text { inhibitory lobe }\end{array}$ & yes & ++ \\
\hline Bowen (1989) & $\begin{array}{l}\text { High- } \\
\text { contrast } \\
25^{\prime} \text { disk }\end{array}$ & $\begin{array}{l}\text { Looked at ratio of peaks in sensory } \\
\text { response function to predict } \\
\text { number of perceived flashes }\end{array}$ & $\begin{array}{l}\text { Multiphasic, } \\
\text { damped } \\
\text { sinusoid }\end{array}$ & yes & ++ \\
\hline $\begin{array}{l}\text { Ohtani \& } \\
\quad \text { Ejima (1988) }\end{array}$ & Gratings & $\begin{array}{l}\text { Adopted Watson's PST model, } \\
\text { inferring characteristics of } \\
\text { low-pass filter under } \\
\text { different stimulus characteristics }\end{array}$ & Biphasic & yes &,,,+++--+-- \\
\hline $\begin{array}{l}\text { Busey \& } \\
\quad \text { Loftus (1994) }\end{array}$ & Digits & $\begin{array}{l}\text { Assumed threshold: } \\
\text { information extracted at some } \\
\text { rate once sensory response } \\
\text { function exceeds threshold }\end{array}$ & Monophasic & no & ++ \\
\hline Smith (1995) & $\begin{array}{l}\text { Squares } \\
\text { presented } \\
\text { in static } \\
\text { noise }\end{array}$ & $\begin{array}{l}\text { Fit reaction time data with a } \\
\text { model assuming contributions } \\
\text { from transient and sustained } \\
\text { mechanisms }\end{array}$ & Biphasic & yes & + \\
\hline
\end{tabular}

Note -+ , positive-contrast pulse; - , negative-contrast pulse.

George Sperling (Sperling \& Sondhi, 1968), and others working in the temporal domain of perception.

The form of the impulse response function, $g(t)$, is formulated as the difference of two gamma functions, the first of which is large and excitatory and the second of which is smaller and inhibitory (Watson, 1986):

$$
g(t)=\frac{(t / \tau)^{n_{1}-1} e^{-t / \tau}}{\tau\left(n_{1}-1\right) !}-s\left[\frac{(t / r \tau)^{n_{2}-1} e^{-t / r \tau}}{r \tau\left(n_{2}-1\right) !}\right],
$$

where $\tau$ represents the time-constant of the gamma function, $n_{1}$ and $n_{2}$ represent the number of exponentially de- caying stages in each gamma function, $r$ represents the delay of the inhibitory component relative to the initial excitatory component, and $t$ represents time since stimulus onset. The amount of temporal inhibition is determined by the contribution of the second term, as determined by the parameter $s$. When $s>0$, this produces a biphasic impulse response function, which is shown as the dashed curve in the middle panel of Figure 1. The left panel of Figure 2 shows two impulse response functions produced by setting $s$ to zero or greater than zero. The current version of the LST model assumes a monophasic impulse response function, which sets $s=0$ and there- 


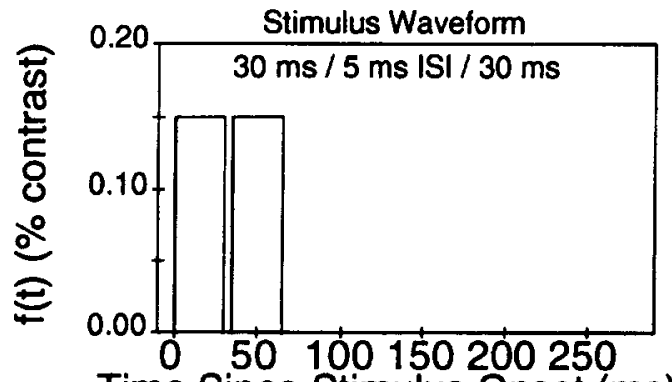

Time Since Stimulus Onset (ms)

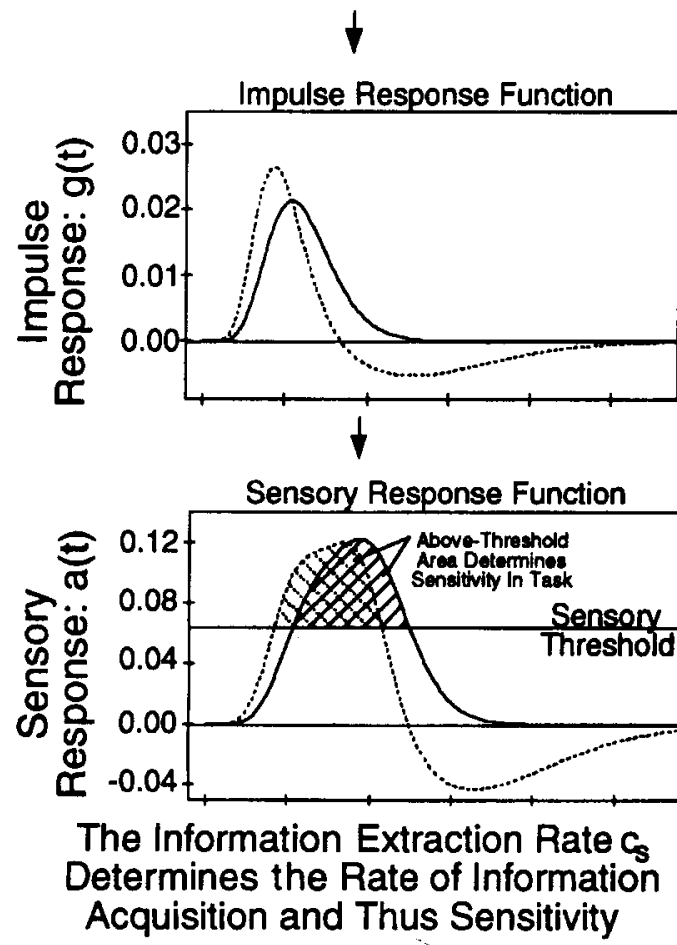

The Physical Stimulus

\author{
Characterization \\ of the Temporal \\ Properties of the \\ Visual System
}

\section{The Visual System's \\ Response to the Physical Stimulus}

Figure 1. Theoretical components of the linear systems filter model of character identification. Top panel: A stimulus is characterized as its contrast over time, in this case a digit appearing for $30 \mathrm{msec}$ at $15 \%$ contrast, disappearing for $5 \mathrm{msec}$, and then reappearing for $30 \mathrm{msec}$ in the same location and at the same contrast. Middle panel: The temporal response properties of the visual system are characterized by an impulse response function that determines how long a brief stimulus persists in time. Temporal inhibition produces a biphasic impulse response function (dashed curve). Bottom panel: The stimulus engenders a response in the visual system that is a function of the stimulus input function $f(t)$ and the impulse response function $g(t)$. The dashed function demonstrates the response produced in a system that includes temporal inhibition. One consequence of the information extraction components of the theory is that performance (or contrast sensitivity) is proportional to the above-threshold area under the sensory response function.

fore includes just the first term of Equation 2, as shown by the solid curve in the middle panel of Figure 1. When $s=0$, the model does not include temporal inhibition.

Detection data for stimuli such as high spatial frequencies and color are often modeled by setting $s$ to zero, which gives a monophasic impulse response function $g(t)$, shown as a solid curve in the left panel of Figure 2 . An alternative way of representing the same information is by taking the Fourier transform of the impulse response function $g(t)$, which results in the transfer function, $G(t)$, which is analogous to the temporal contrast sensitivity function (TCSF). The TCSF plot shows the sensitivity of a system to different temporal frequencies. The TCSF corresponding to the solid line in the left panel of Figure 2 is given by the solid line in the right panel. These curves represent a purely sustained response and give a monotonically decreasing TCSF.

Detection data for stimuli containing mainly low spatial frequencies, or stimuli presented on bright backgrounds, often are modeled by $s>0$. In this case, the impulse re- 

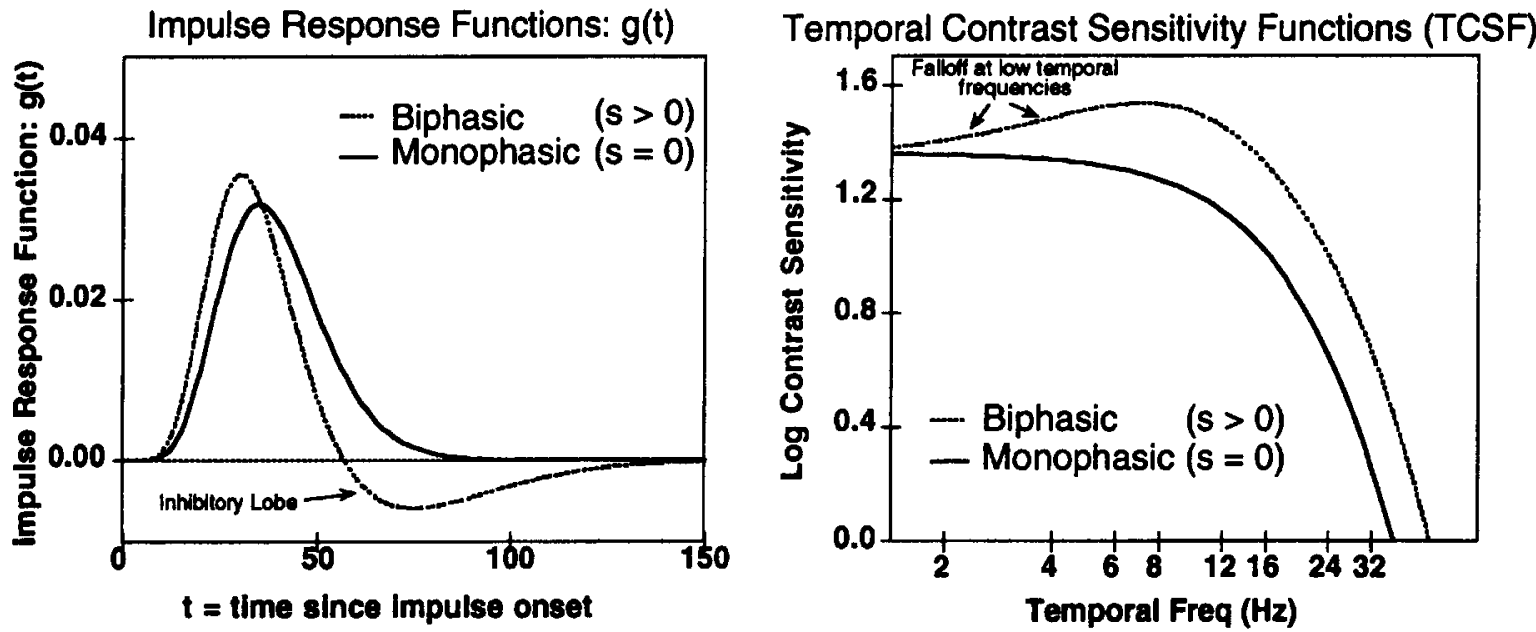

Figure 2. The temporal frequencies underlying a task may be characterized by an impulse response function (left panel) that characterizes the time course of the perceptual response engendered by a stimulus, or by the temporal contrast sensitivity function (TCSF; right panel), which characterizes the fidelity by which the pathways subserving a given task pass different temporal frequencies. A monophasic impulse response function has no inhibitory lobe, no dip in the TCSF at low temporal frequencies, and by definition no temporal inhibition. A biphasic impulse response function includes all three. Parameters used: monophasic, $\tau=4.4, s=0\}$; biphasic, $\{\tau=3.58, r=2.0, s=0.39\}$.

sponse inhibits processing after an initial excitatory response, which results in an inhibitory lobe in the impulse response function $g(t)$ (dashed line in Figure 2, left panel) and a characteristic TCSF with a decrease in sensitivity at low temporal frequencies (dashed curve in Figure 2, right panel). This decrease is caused by the inhibitory lobe and causes the system to inhibit itself prematurely. for slowly changing stimuli.

Information loss: The sensory threshold. All linear filter models include some form of nonlinearity after the initial linear filter portions. The LST model has adopted a sensory threshold (Busey \& Loftus, 1994; Loftus et al., 1993), so that only that portion of the $a(t)$ function that rises above the threshold contributes to performance. In addition, because information relevant to character identification is present in both positive- and negative-contrast pulses, we take the absolute value of the signal after assessing the sensory threshold. Mathematically, this is expressed as

$$
a_{\theta}(t)=\left\{\begin{array}{cll}
|a(t)-\theta| & \text { if } & a(t)>\theta \\
0 & \text { if } \theta>a(t)>-\theta \\
|a(t)+\theta| & \text { if } a(t)<-\theta
\end{array}\right\} .
$$

This rectification simply states that information can be acquired from both positive- and negative-contrast stimuli, not that observers cannot tell the difference between a light and dark digit. ${ }^{1}$ The exact form of the sensory nonlinearity is not critical: Watson's probability summation in time model (Watson, 1978), which uses a power function as a nonlinearity, will make equivalent predictions. The power function acts very similarly to a hard threshold, since they both tend to punish weak responses and reward strong responses. For example, a long dim stimulus may never rise above threshold and never produce a response; likewise, the same stimulus will produce a very small response in Watson's (1978) model, since when the $a(t)$ values are raised to an exponent, small numbers become much smaller while larger numbers (close to 1.0 ) remain relatively unchanged or even become larger. Thus both models selectively impair the responses to weak signals.

Note that the sensory threshold, $\theta$, is conceptually quite different from a detection threshold, which is a statistical concept representing a certain performance level. $\theta$ is also distinct from the decision thresholds used in detection linear filter models, which assume that the stimulus is detected if some representation within the model exceeds the detection threshold.

Acquired information: The information extraction rate. Once the sensory response function $a(t)$ exceeds the sensory threshold $\theta$ (as implied by $a_{\Theta}(t)>0$ ), information is extracted at some acquisition rate. This function represents the instantaneous rate of information acquisition and is proportional to (1) the above-threshold sensory response and (2) the proportion of remaining, to-be-acquired, stimulus information. The first part of this expression, the above-threshold sensory response, is given by Equation 3 . For the second half of the expression, define $I(t)$ as the proportion of information acquired by time $t$. The proportion of remaining stimulus information is simply $[1.0-I(t)]$. The derivative of $I(t)$ with respect to time is defined as

$$
\frac{d I}{d t}=a_{\Theta}(t)\left[\frac{1.0-I(t)}{c_{s}}\right]
$$

where $c_{s}$ represents the constant of proportionality and completes the equality. The model parameter $1 / c_{s}$ also 

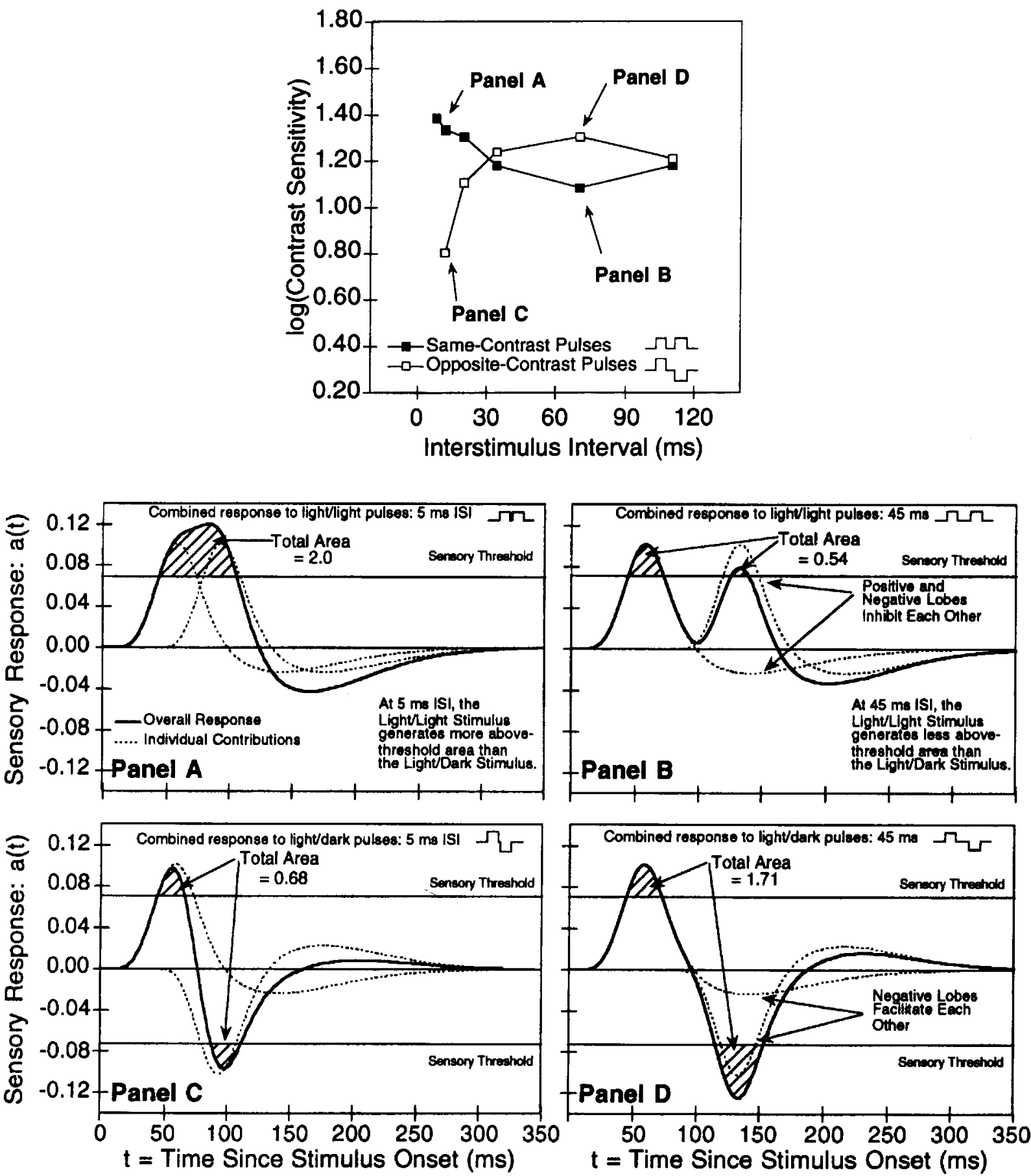

Figure 3. Two-pulse detection data adapted from Rashbass (1970). The linear systems theory model (Busey \& Loftus, 1994) can account for temporal inhibition seen in these data when extended with a biphasic impulse response function via Equation 2 . Contrast sensitivity is directly related to the above-threshold area. As interstimulus interval increases, sensitivity decreases in the samecontrast condition (panels $A$ and $B$ ) as the inhibitory lobe from the first pulse inhibits the excitatory portion of the response to the second pulse (panel B). Sensitivity increases in the opposite-contrast condition (panels $C$ and $D$ ) since the inhibitory lobe from the first pulse facilitates the negative-going excitatory lobe from the opposite-contrast second pulse (panel D). 
represents the rate at which new (i.e., previously unsampled) features are sampled, although the overall rate of feature acquisition decreases over time as $I(t)$ rises (since $1.0-I(t)$ decreases).

Busey and Loftus (1994) demonstrated that with this rate function the equation relating total acquired information, which we designate $I(\infty)$, to the above-threshold area under $a_{\Theta}(t), A_{\Theta}(\infty)$, becomes

$$
I(\infty)=1.0-e^{-A_{\Theta}(\infty) / c_{s}}
$$

Performance predictions. In order to make quantitative predictions, the theory requires one additional linking assumption. Total acquired information $I(\infty)$ is assumed to equal $p$, the proportion of correctly recalled digits. Thus,

$$
p=1.0-e^{-A_{\Theta}(\infty) / c_{s}} .
$$

Equation 6 summarizes an important predictionthat performance is directly related to the above-threshold area under the $a(t)$ function. This relationship is a consequence of Equations 1, 3, 4, and 5 and is not an assumption of the theory.

Accounting for two-pulse detection data: The role of temporal inhibition. Equation 6 can be used to make predictions for contrast threshold data, which is common in detection experiments. In this case, $p$ is fixed at the performance threshold (usually $75 \%$ correct detection), and the predicted contrast $\Phi$ is systematically varied to produce a predicted performance near $75 \%$ for a given set of parameters $(\tau, r, s$, and $\theta)$. A condition that produces more above-threshold area (e.g., a longer stimulus duration) will require a smaller contrast to keep performance at $75 \%$ correct. Thus contrast threshold is proportional to the above-threshold area.

Figure 3 plots two-pulse data from Rashbass (1970), along with a description of how the LST model, modified for temporal inhibition via Equation 2, accounts for the crossover in sensitivity that occurs between the light/ light and light/dark conditions as ISI increases. For a 5 -msec ISI, sensitivity is quite good for the light/light condition, but decreases with increasing ISI. This results both from the separation of the two pulses pushing less area about the sensory threshold and the inhibitory lobe from the first pulse reducing the excitatory lobe from the second pulse. Both of these result in less area above threshold and thus predict a decrease in sensitivity as ISI increases by Equation 6 .

The opposite situation exists for the light/dark stimuli. Sensitivity is poor at very short ISIs since the two pulses tend to cancel each other out. However, as the ISI is increased, the inhibitory negative lobe from the first pulse facilitates the negative-going excitatory lobe from the second pulse, which is assumed to be negative going since it has a negative contrast relative to the first pulse. The facilitation between the two negative-going lobes increases the above-threshold area and thus improves the overall sensitivity to the light/dark stimulus at intermediate ISIs by Equation 6 .

\section{Empirical Evidence for Inhibition in Character Detection and Identification Tasks}

The picture that emerges from the two-pulse detection literature is fairly clear: The temporal inhibitory mechanisms depend on the adaptation level as well as on stimulus size. Stimuli that are shown on adaptation levels higher than about $5 \mathrm{~cd} / \mathrm{m}^{2}$ and are larger than about 13' of arc (or containing spatial frequencies larger than .751.5 cycles per degree) show evidence of producing inhibitory responses. Delay intervals between the two pulses of about $40-70 \mathrm{msec}$ provide the best evidence for temporal inhibition. The two-pulse paradigm with both positiveand negative-contrast pulses is perhaps the best paradigm for demonstrating evidence for temporal inhibition.

The goal of the three experiments discussed below is to test these questions: Do character detection and identification tasks show evidence of temporal inhibition that is consistent with the two-pulse detection literature? If so, can the character identification model of Loftus and Busey (Busey \& Loftus, 1994; Loftus et al., 1993) be extended to account for this temporal inhibition? Experiment 1 generalized detection tasks to digit stimuli, using a task in which digits are the to-be-detected stimuli, to verify that temporal inhibition exists for the detection of characters presented at threshold contrasts. Experiment 2 was a traditional character identification task that examined whether temporal inhibition exists in a digit recall task under suprathreshold conditions. Experiment 3 measured the range of temporal frequencies that contribute to character identification, using digits flickered at different temporal frequencies. Experiment 1 focused more on the initial visual processing stages because the identity of the digit is not important. Experiment 2 then examined the temporal inhibition present when the task requires identification of the digit. Experiment 3 provided converging evidence for Experiment 2 using a different paradigm that discriminates between candidate neural models of temporal inhibition.

\section{EXPERIMENT 1 \\ Threshold Detection of Pulsed Digits}

To investigate whether character stimuli show the same patterns of temporal inhibition, a single digit was presented for two pulses. This two-pulse stimulus could appear in either of two temporal intervals delimited by low tones. Observers made a two-alternative-temporal forced choice (2ATFC) response regarding the interval that contained the stimulus. Four types of conditions were used: ,,+++--+ , and -- , where + indicates a positive-contrast pulse and - indicates a negative-contrast pulse. The second pulse was delayed for one of six intervals ranging from 0 to $120 \mathrm{msec}$. The pulses were presented in one 

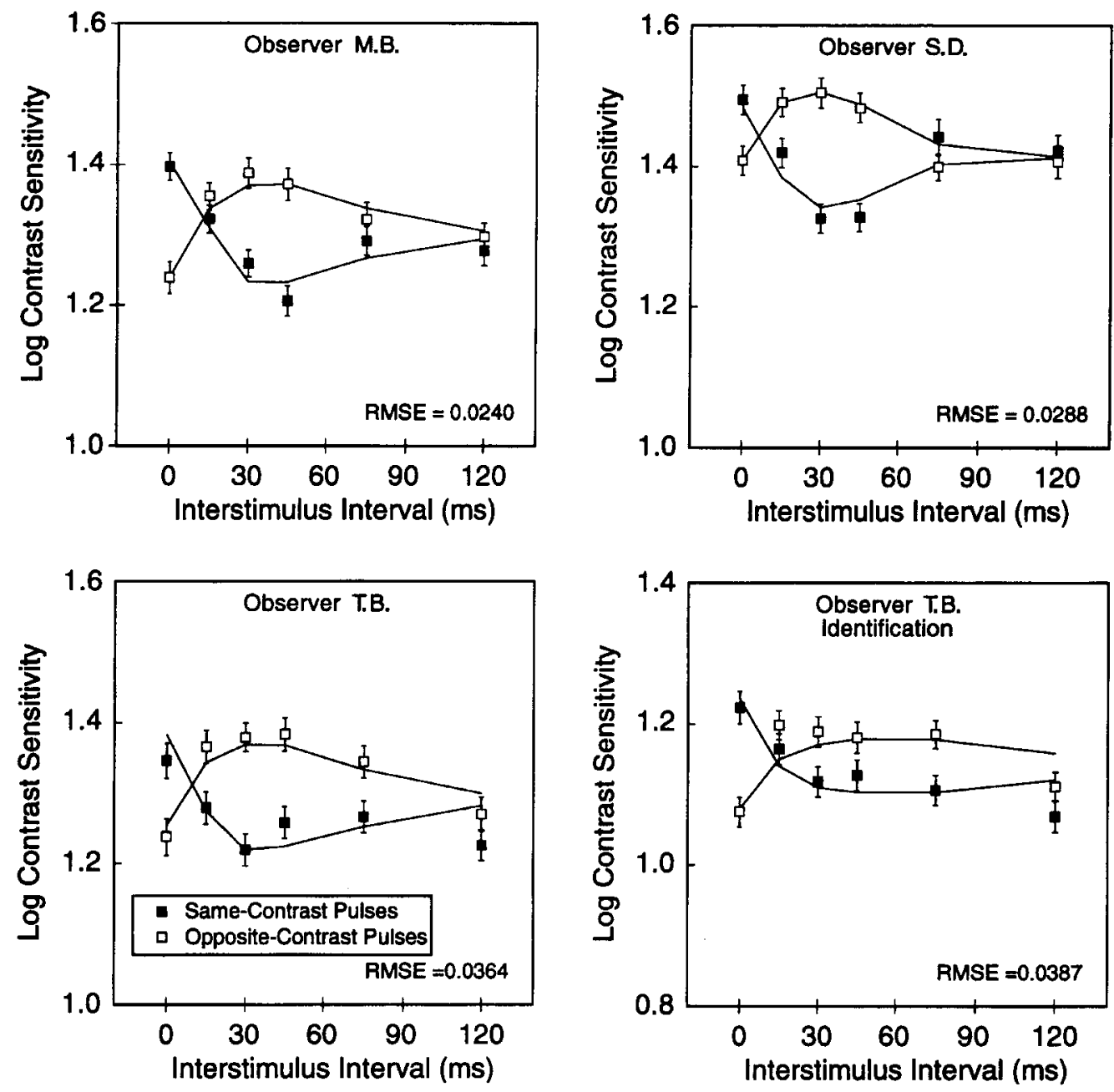

Figure 4. Experiment 1 results for 3 observers. Conditions are averaged across stimulus type, so that the ++ and - conditions are shown as the same-contrast case, and the -+ and +- conditions are shown as the opposite-contrast case. Error bars represent the standard deviation of the threshold-finding function. The lower right panel shows the data and model fit for Observer T.B. for an identification task, which is described in the text. Error bars represent $1 S D$ of the QUEST function (Watson \& Pelli, 1983).

of two temporal intervals, and the subject had to identify the correct interval; this made the identity of the digit irrelevant. The contrast of the digits was manipulated to determine the contrast that yielded $82 \%$ correct detection of any digit. I then modeled these contrast thresholds with the theory to see if the linear filter model of Loftus and Busey could account for the obtained same-contrast and different-contrast thresholds.

\section{Method}

Stimulus presentation and response collection were carried out on a Macintosh II computer.

Observers. Three observers - the author (T.B.) and 2 male graduate students (S.D. and M.B.)-participated in Experiments 1 and 2. All observers had participated in a minimum of 1,000 trials prior to participating in Experiment 1.

Stimuli and Apparatus. The experiment was controlled by a Macintosh II computer, and stimuli were presented on an Apple
Monochrome monitor with a Video Attenuator (Pelli \& Zhang, 1991) to allow a greater range of luminance values. The screen was calibrated using a Minolta Spotmeter LS1 10 and the calibration techniques that are part of the VideoToolbox library (Pelli, 1997). Observers sat approximately $57 \mathrm{~cm}$ away from the screen in a dimly lit room and used the computer keypad to respond. The refresh rate of the monitor was $67 \mathrm{~Hz}$

The background luminance was set to $7.2 \mathrm{~cd} / \mathrm{m}^{2}$, and the fixation point had a luminance of $3.7 \mathrm{~cd} / \mathrm{m}^{2}$. Contrast was defined as (pulse luminance - background luminance)/(pulse luminance + background luminance).

The digits were either a 2 or a 5, drawn in Times Roman 14-point font. The digits were each $0.50^{\circ}$ high $\times 0.40^{\circ}$ wide and always appeared centered vertically on the screen. The top portion of the digit was $0.27^{\circ}$ below the fixation point.

Design. In all trials, the duration of each of two pulses was $30 \mathrm{msec}$ (two screen refreshes). The two pulses were separated by one of six delays, ranging from 0 to $120 \mathrm{msec}$, during which the screen was the background luminance. The two pulses were always of the same magnitude, although they may have been in different direc- 
Table 3

Summary of Best-Fitting Model Parameters for Experiments 1-3

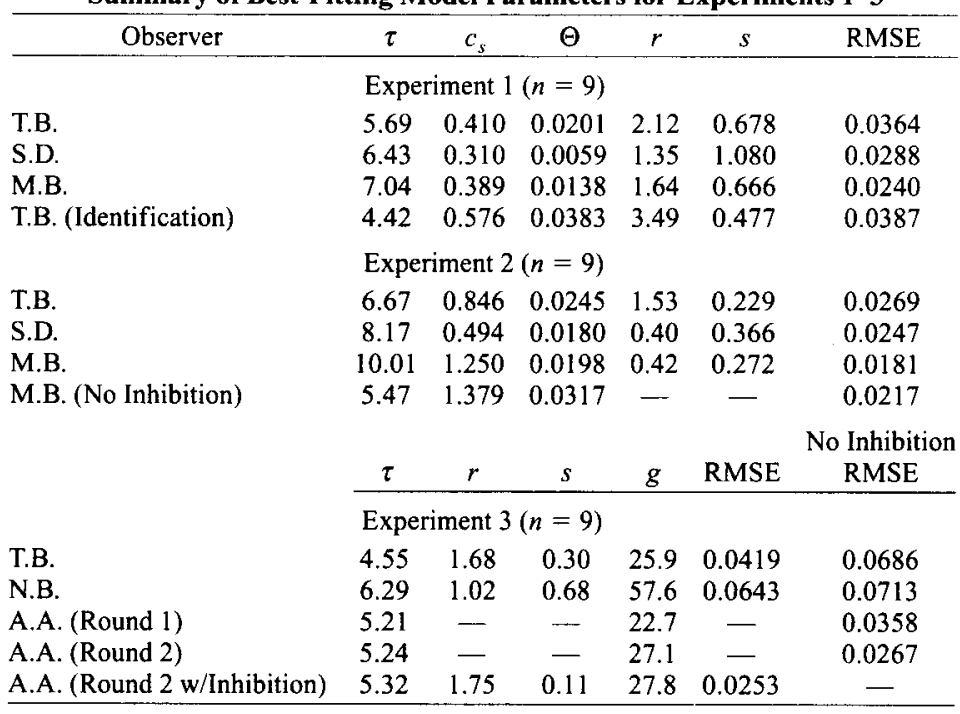

Note-RMSEs are in units of $\log (1 /$ contrast $)$ for Experiments 1 and 3, and units of proportion correct for Experiment 2. For Experiment 3, no inhibition RMSE.

tions (positive or negative contrast) depending on the stimulus condition.

Observers completed 25 blocks of 96 trials each, which provided 100 observations per condition per observer.

Procedure. A trial consisted of two temporal intervals separated by low tones, each of which contained the stimulus with $50 \%$ probability. After the second interval, observers indicated which of the two intervals they thought contained the digit, guessing if necessary. They then received feedback in the form of a tone.

An extension of this experiment was conducted with Observer T.B. in which the identity of the digit, which was either 2 or 5 , was the relevant stimulus attribute.

An adaptive threshold finding procedure, QUEST (Watson \& Pelli, 1983), was used to adjust the contrast of the pulses to find the contrast that provided an $82 \%$ detection rate. This value optimizes the efficiency of the QUEST threshold-finding procedures. The QUEST procedure presents the stimulus at various contrasts and uses the resulting detection performance to estimate the contrast threshold. Detection performance at all contrasts is used to estimate the contrast threshold.

\section{Results and Discussion}

Figure 4 shows the results of 3 observers for Experiment 1; the smooth curves through the data points are explained below. For an ISI of zero, ${ }^{2}$ observers were more sensitive to the same-contrast condition than the oppositecontrast condition, which is indicated by the lower threshold for the former condition. However, when a small ISI of $15-30 \mathrm{msec}$ was introduced, this pattern reversed and all 3 observers became more sensitive to the opposite-contrast condition than to the same-contrast condition. These differences between the two conditions grew largest at 30 - to 45-msec ISIs. At the empirical level, these results indicate that digit stimuli show the same kinds of detection curves that others have reported for simpler stimuli such as disks or gratings. Thus it appears that the detection of digit stimuli is similar to the detection of disks and gratings.

At the theoretical level, these findings clearly implicate temporal inhibition, and the results are quite similar to those of Ohtani and Ejima (1988). For comparison with their results, our background level of $7.272 \mathrm{~cd} / \mathrm{m}^{2}$ translates to about $90 \mathrm{td}$, which is in the mesopic range.

Temporal inhibition as revealed by this task begins at delay intervals of 15-30 msec and extends to $45-70 \mathrm{msec}$ for the 3 observers. This is consistent with the ranges given in Table 1, which show temporal inhibition for delay intervals of 30-70 msec for a variety of display conditions.

Differences across conditions. The results in Figure 4 are averaged across the sign of the contrast of the stimuli $(++$ and -- were averaged together, as were +- and -+ ). Consistent with previous findings (Blackwell, 1963; Boynton, 1972; Rashbass, 1970), no systematic differences were found between the individual conditions. The exception was Observer S.D., who had small but systematic deviations between the ++ and - - contrast thresholds. He was more sensitive to the -- condition, which had a dark character on a gray background. However, these deviations were consistent across ISI, and thus the average does not distort the overall pattern of the contrast thresholds.

Identification task. For direct comparison with the detection task in Experiment 1, identification data were collected for Observer T.B. The stimuli were either a 2 or a 5 , and the contrast of the digits was systematically adjusted until identification performance reached $81 \%$. The stimuli were presented in a single temporal interval; display conditions were otherwise identical to those of Experiment 1. 
The obtained identification contrast sensitivity values are shown in the lower right panel of Figure 4, along with the corresponding model fit, which includes a biphasic impulse response function. For Observer T. B., the effects of temporal inhibition seem to have existed for slightly longer display intervals in the identification task (see Figure 4, lower right panel) $(45-70 \mathrm{msec})$ than in the detection task (30-70 msec). In addition, the size of the temporal inhibition appears to have been slightly smaller for the identification task. In general, these data are not much different from the detection data, although the overall sensitivity values differ (note the different ordinate scales).

Model predictions. The linear filter model of character identification was applied to the data in Figure 4. Following Busey and Loftus (1994, Experiment 6), the theory may be extended to detection tasks by assuming that detection performance is proportional to the above- threshold area under the sensory response function. This theory, which assumes a monophasic impulse response function and therefore no temporal inhibition, is clearly disconfirmed by the evidence for temporal inhibition seen at 45- to 60-msec ISI ranges in all 3 observers. However, the model may be extended to account for these data by adopting Watson's (1986) working model's characterization of the impulse response function (see Equation 2). The best-fitting predictions for the revised model are shown in Figure 4. These fits are all quite acceptable, except perhaps for Observer T. B. at a 120 -msec ISI. The parameter values for each fit, given in Table 3, are all in reasonable ranges.

Experiment 1 clearly demonstrates evidence for temporal inhibition in a detection task in which characters are used as stimuli. This is true even when the identity of the digit becomes important, as with Observer T.B. These results indicate the need for a model that assumes tem-
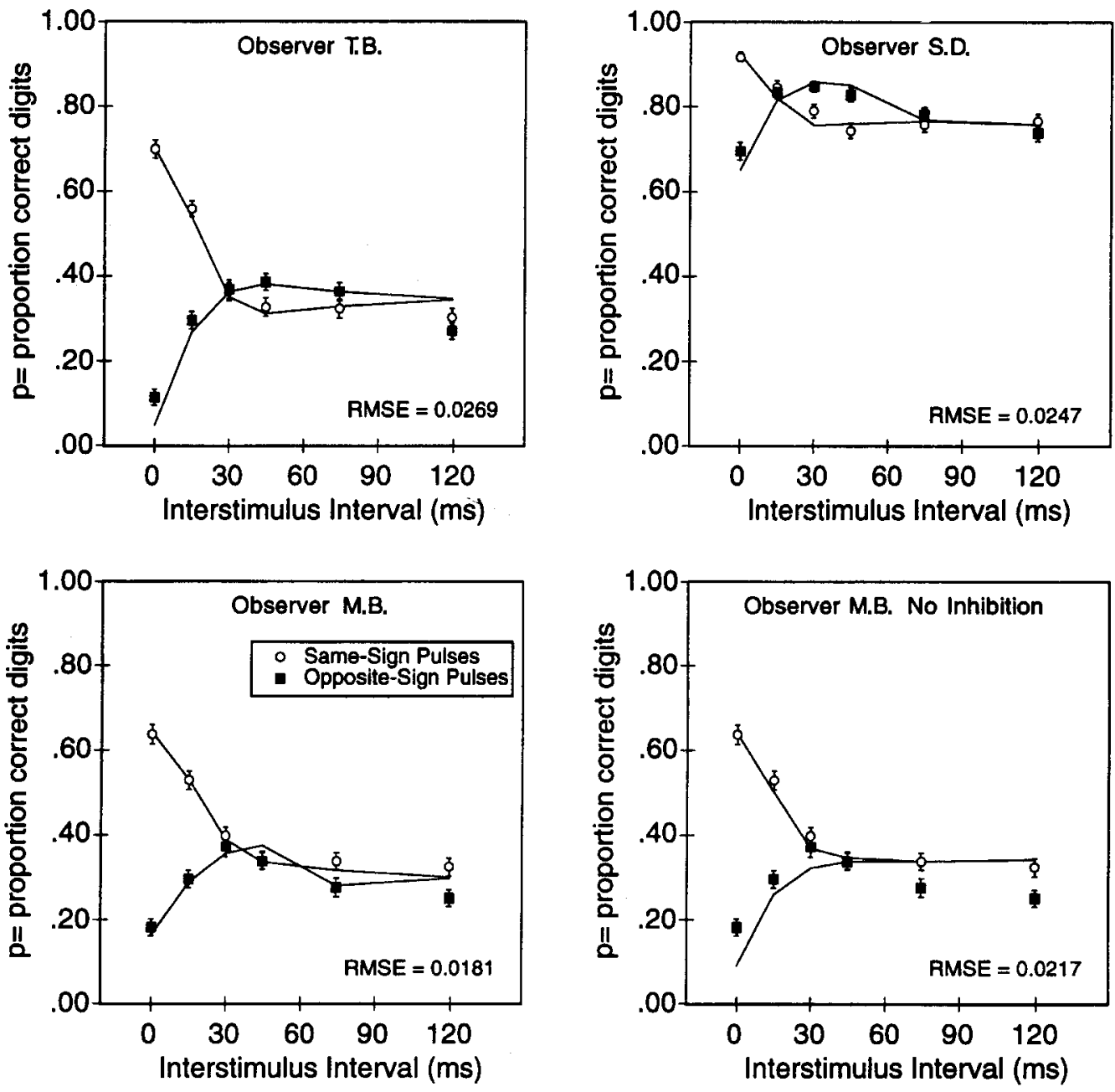

Figure 5. Experiment 2 results for 3 observers. Background luminance was identical to that in Experiment 1. Task was recalling four digits. Error bars represent $1 S E M$. The predictions for the modified character identification theory are shown as the curves. The lower right graph shows the relatively poor fit of the noninhibition model to Observer M.B.'s data. Error bars represent 1 SEM. 
poral inhibition, and when the character identification theory is modified to include this component it provides good predictions for the detection data.

\section{EXPERIMENT 2 Identification of Different-Contrast Pulsed Digits}

Experiment 1 verified that digit stimuli in a threshold detection task exhibit the same pattern of temporal summation under the same background luminance levels as more simple stimuli such as disks or gratings. For the most part, however, the actual identity of the digit was not perceptible; observers merely had to indicate which temporal interval contained any contrast change. Therefore, Experiment 1 did not call upon the cognitive mechanisms that are involved in recognizing and remembering the identity of the digits. Experiment 2 was designed to introduce these cognitive mechanisms to see how temporal inhibitory processes might influence these processes at suprathreshold contrast levels. This experiment also provided a direct test of the linear filter model of character identification of Busey and Loftus (1994), which did not assume temporal inhibition.

The task in Experiment 2 was a common information processing task in which four digits are presented to an observer whose job is to report as many of them as possible, in their correct order, guessing if necessary. The basic performance measure, $p$, is the proportion of correctly reported digits, in their correct locations, adjusted for the guessing probability. ${ }^{3}$

\section{Method}

Stimulus presentation and response collection were identical to those in Experiment 1. In Experiment 2, observers viewed twopulse presentations of four digits. Their task was to perceive the digits and type them into a computer keypad. The same durations and delays as those used in Experiment 1 were employed.

Observers. Three observers - the author (T.B.) and 2 male graduate students (S.D. and M.B.) - participated in both Experiments 1 and 2. All observers had participated in a minimum of 1,000 trials prior to participating in Experiment 1.

Stimuli and Apparatus. The background luminance was again set to $7.272 \mathrm{~cd} / \mathrm{m}^{2}$, and the fixation point had a luminance of $3.7 \mathrm{~cd} / \mathrm{m}^{2}$. For 2 observers (S.D. and T.B.), the contrast of the digits was either $6.5 \%$ or $-6.5 \%$, depending on the nature of the condition. A 3rd observer (M.B.) had contrasts of $7 \%$ and $-7 \%$. Contrast was defined as (foreground luminance - background luminance)/(foreground luminance + background luminance).

The stimuli were adapted from those used in related information processing tasks (e.g., Sperling, 1960) and consisted of 12 digits arranged in three rows. Observers reported the contents of one row of 4 digits in order, guessing if necessary. In this task, unlike partial report tasks, the observer knew in advance which row to attend to, which was the same for all trials in a block. The use of three rows was maintained for consistency with previous information processing experiments (e.g., Busey \& Loftus, 1994; Loftus et al., 1993). To avoid masking effects from the fixation point, only the top and the bottom rows were used. The digits were each $0.50^{\circ}$ high $\times 0.40^{\circ}$ wide, and the edges of each digit were separated by $0.75^{\circ}$ vertically and $0.40^{\circ}$ horizontally. This stimulus configuration implies eccentricity differences between the stimuli in Experiment 1 and 2 . However, in both experiments, the observer was free to fix- ate on any portion of the display deemed most productive. The observers reported looking at a point in between positions 2 and 3 . The entire row of digits spanned $0.50^{\circ}$ vertically and $2.8^{\circ}$ horizontally.

Design. In all trials, the duration of each of two pulses was $30 \mathrm{msec}$ (two screen refreshes). The two pulses were separated by one of six delays, ranging from 0 to $120 \mathrm{msec}$, in which the screen was the background luminance. Observers completed 24 blocks of 72 trials each, which provided 72 observations per condition per observer.

Procedure. A trial began with a $250-\mathrm{msec}$ warning tone that reminded observers of the to-be-reported row (either the top or the bottom row) for the current block of trials. Following the tone, the digit array was displayed in its appropriate temporal configuration, followed $375 \mathrm{msec}$ later by a response tone. The observer then tried to type the digits in their proper order, guessing if necessary. The observers then received feedback in the form of four tones.

\section{Results and Discussion}

Figure 5 shows the results for the 3 observers in Experiment 2 . For a $0-\mathrm{msec}$ ISI condition, performance was much worse in the opposite-contrast conditions, but gradually increased as ISI increased. At a $45-\mathrm{msec}$ ISI, opposite-contrast performance exceeded same-contrast performance for 2 observers (T.B. and S.D.), which is consistent with the results reported in the two-pulse detection literature and Experiment 1 . The 3rd observer (M.B.) did not show this reversal.

At a theoretical level, the data from Observers T.B. and S.D. clearly indicate effects of temporal inhibition.

Evidence for temporal inhibition as revealed by this digit recall task occurs at delay intervals of $30-45 \mathrm{msec}$ for Observers T.B. and S.D. The size of these inhibitory effects was smaller than that for the detection data. This is consistent with the Experiment 1 findings for $\mathrm{Ob}$ server T.B., whose inhibitory effect was smaller in the identification task than in the detection task. However, as in Experiment 1, the range of empirically determined temporal inhibitory effects is consistent with that reported in Table 1, suggesting that the temporal processing of character information and simpler stimuli such as disks and gratings follows similar mechanisms.

Differences across conditions. As in Experiment 1, no systematic differences between the ++ and -- or -+ and +- conditions were observed. However, as in Experiment 1 , the sole exception was Observer S.D., who had small but systematic deviations between the ++ and - - contrast thresholds. Consistent with his Experiment 1 contrast thresholds, he performed better in the -- condition than in the ++ condition. However, these deviations were again consistent across ISIs, and thus the average does not distort the overall pattern of the obtained performance levels.

Observer T.B. showed consistent deviations from the model predictions at the 120-msec ISI for both Experiments 1 and 2. The reasons for these are not clear. The long ISI could have provided an opportunity for eye movements to intervene. This would have caused the second presentation to appear on a different portion of the retina and therefore not sum completely with the first pulse. 
Model predictions. Model predictions for Experiment 2 were generated using the LST model, modified to include temporal inhibition. These predictions are shown as curves in Figure 6 . The need for an impulse response function with an inhibitory lobe is clearly indicated by the fact that performance at the $45-\mathrm{msec}$ delay was superior in the opposite-signed condition than in the samesigned condition. Observers T.B. and S.D. demonstrated this pattern, although observer M.B. did not. Figure 4 demonstrates how temporal inhibition accounts for this data pattern, where more above-threshold area produces a prediction of better performance.

The best-fitting model parameters for these fits are given in Table 3 . In general, the fits are quite good, with the exception of a slight underprediction for Observer T.B. at the short ISI for the opposite-contrast condition, and a slight overprediction for the long ISI condition for the same observer. The slight performance decrement below the probability summation prediction for the 120 msec ISI for Observer T.B. is consistent with the slightly higher contrast thresholds at the same ISI for this observer in Experiment 1.

Observer M.B. did not show any temporal inhibition at any ISI delay, as indicated by the criterion of oppositecontrast performance greater than same-contrast performance. Therefore, a version of the character identification theory that does not assume temporal inhibition might fit these data. Such a fit was attempted and parameter values are given in Table 3, but the fit was quite poor, as evidenced by the graph in the lower right panel of Figure 5 and the larger root-mean-squared error given in Table 3 for this fit relative to the version that included temporal inhibition.

The model fits shown in Figure 5 are fairly close to the data, but for 2 of the 3 observers, they were obtained using model parameters that are, on the surface, perplexing. For Observers M.B. and S.D., the ratio of the inhibition $\tau$ to the excitatory $\tau$ was less than 1.0. This implies an impulse response function with an initial small negative lobe and then a much larger positive lobe. Although most models of two-pulse data have an initial excitatory lobe, both Roufs and Blommaert (1981) and den Brinker (1989) consider impulse response functions with initial inhibitory lobes. However, two-pulse thresholds do not contain phase information, and therefore will be equally well fit by a time-reversed impulse response function (Watson, personal communication, October 30, 1995). In this case, a small negative lobe followed by a large positive lobe is equivalent to a large positive lobe followed by a small negative lobe. Thus exactly equivalent fits could be obtained by restricting $r$ to be greater than 1.0 in the case of Observers M.B. and S.D.

Effect of background luminance. One issue not addressed by the Experiment 2 data is the dependence of temporal inhibition on the background luminance seen in Table 1. This is particularly important for characters since they are often viewed in high-luminance conditions, which may affect the amount of temporal inhibi- tion. Ikeda (1986) and others have pointed out the dependence of temporal inhibition on the background luminance level, and this dependence was verified with Observers M.B. and T.B. in several small experiments. These 2 observers were retested using the Experiment 2 paradigm at the $45-\mathrm{msec}$ ISI delay, which was chosen because it gives the most evidence for temporal inhibition. The background luminance was raised by $0.5 \log$ units, to $11.7 \mathrm{~cd} / \mathrm{m}^{2}$, and the contrast was decreased to $5 \%$ for Observer M.B. and 4.5\% for Observer T.B. to keep performance off the ceiling.

Observer M.B., who did not show evidence of temporal inhibition at the $7.272 \mathrm{~cd} / \mathrm{m}^{2}$ background level, showed strong evidence for temporal inhibition at this higher background level. Opposite-contrast performance was 0.660 , while same-contrast performance was 0.501 . Observer T.B. showed the same pattern; opposite-contrast performance was 0.437 , while same-contrast performance was 0.309 . The standard error for each mean was about 0.029 . Two-tailed $t$ tests were significant for both observers at $\alpha=.05$, demonstrating a pattern that is consistent with the detection literature and that implies temporal inhibition at a theoretical level.

According to the results summarized in Table 1, lower background luminance levels do not produce temporal inhibition. To verify this for the character identification task, data were collected from Observer T.B. at the 45msec ISI delay condition for a background level that was $0.5 \log$ units lower in luminance $\left(1.17 \mathrm{~cd} / \mathrm{m}^{2}\right)$ than Experiment 2's background luminance. Contrast was set at $9 \%$ to raise performance above chance.

As anticipated, this condition produced no evidence for temporal inhibition; indeed, the same-contrast performance $(0.379)$ was actually better than the oppositecontrast performance $(0.306)$. The standard error for each mean was about 0.030 . This pattern is consistent with findings from detection studies at lower background luminance levels in which same-contrast and oppositecontrast performances did not differ. At a theoretical level, this does not imply temporal inhibition.

These results confirm the dependence of temporal inhibition on the background luminance level. Although a complete parametric study was not attempted, these data confirm the finding that background luminance levels around $1 \mathrm{~cd} / \mathrm{m}^{2}$ do not produce temporal inhibition, whereas luminance levels above $7 \mathrm{~cd} / \mathrm{m}^{2}$ do produce temporal inhibition in most observers.

\section{EXPERIMENT 3}

Experiments 1 and 2 provided evidence for temporal inhibition in both detection and character identification tasks when digits are used as stimuli. Several authors have proposed mechanisms that underlie temporal inhibition, and the goal of Experiment 3 was to discriminate between these explanations.

Ikeda (1986) noted the dependence of temporal inhibition on stimulus size and echoed the suggestion of Pur- 

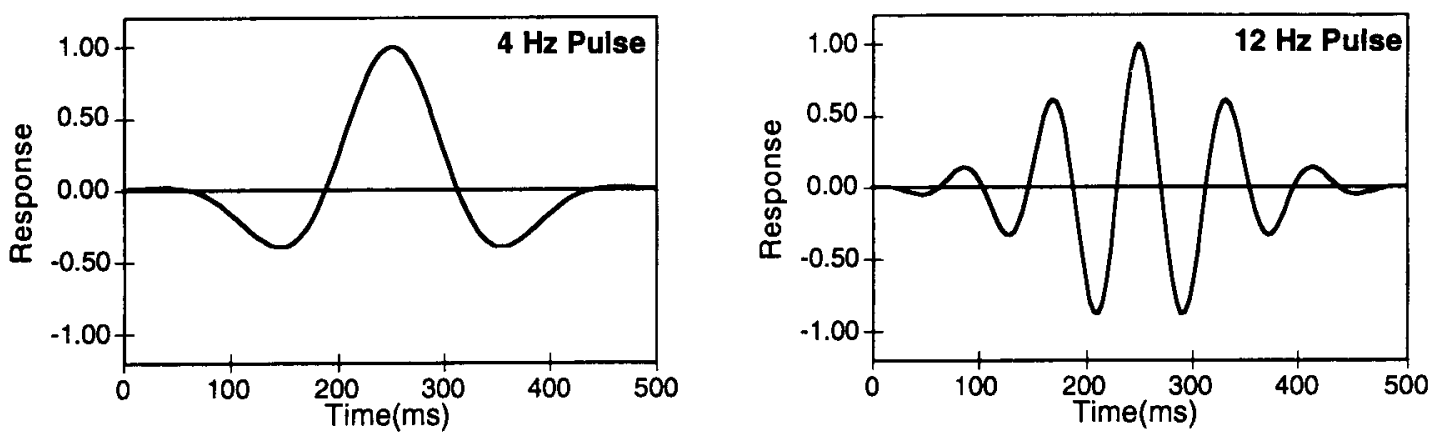

Figure 6. Examples of temporal waveforms used in Experiment 3.

cell and Stewart (1971), who suggested that lateral inhibitory mechanisms in the retina might underlie temporal inhibition. This mechanism was further elaborated in the context of the lateral inhibitory mechanisms associated with center-surround cells in the retina by Enroth-Cugell, Robson, Schweitzer-Tong, and Watson (1983). Under this model, temporal inhibition results from interactions within a channel, in which the surround of a center-surround cell inhibits the excitatory response from the center of the receptive field.

A second mechanism involves transient-on-sustained inhibition, in which the onset or offset of the second pulse generates a rapid transient response that inhibits the slow excitatory processing of the first pulse. This form of inhibition has been used to explain a variety of masking processes (see, e.g., Breitmeyer, 1984). Breitmeyer (1984) also suggested that the abrupt onsets associated with the second pulse of a two-pulse stimulus could generate a fast inhibitory response in the transient channel that inhibits the processing of the first pulse. Thus the acrosschannel inhibitory mechanisms might produce inhibitory effects that are consistent with temporal inhibition. Under this model, the temporal inhibition results from activation of the transient system by the abrupt onset of the second pulse.

Experiment 3 addressed the role of abrupt onsets in temporal inhibition by removing all abrupt onsets from the stimulus and determining whether evidence for temporal inhibition would persist. The task involved flickering a digit at different temporal frequencies and adjusting the contrast of the digit until it was just barely identifiable. Although the task was to identify the digit, the intrinsic temporal properties of the system mediating character identification placed limits on the sensitivity of character identification at different flicker rates. Evidence for temporal inhibition comes from the very slowest flicker rates. Temporal inhibition acts to sharpen the response in the visual system, but for a very slowly flickering stimulus, the system tends to inhibit itself. This results in a decrease in sensitivity at very slow temporal frequencies (e.g., $2 \mathrm{~Hz}$ ) relative to moderate frequencies (e.g., $8 \mathrm{~Hz}$ ), and this characteristic fall-off in sensitivity is a signature of temporal inhibition. When sensitivity measured at differ- ent temporal frequencies is plotted against the flicker rate, a TCSF plot can be used to derive the impulse response function and demonstrate evidence for temporal inhibition. A TCSF plot that includes this fall-off at low temporal frequencies is directly associated with a biphasic impulse response function, as demonstrated by Figure 2 .

Alexander et al. (1994) flickered letters at 2 and $16 \mathrm{~Hz}$ and measured spatial contrast sensitivity functions (SCSF). At the $2-\mathrm{Hz}$ flicker rate, they found that the SCSF differed systematically from a function generated from gratings. Such differences did not exist at the $16-\mathrm{Hz}$ flicker rate. The differences at $2 \mathrm{~Hz}$ demonstrate that temporal frequency has a systematic effect on the relation between contrast sensitivity and stimulus type. Alexander et al. did not construct a full TCSF for their letters.

The TCSF paradigm used in Experiment 3 has several disadvantages compared with the two-pulse paradigm used in Experiments 1 and 2. The primary advantage of the two-pulse stimulus is that it is temporally compact, so the longest stimulus durations are in the range of $120-150 \mathrm{msec}$. Evidence for temporal inhibition in the TCSF paradigm comes from very slow temporal frequencies, necessitating very long $(500-\mathrm{msec})$ stimulus durations. These long durations may allow eye movements, which can improve sensitivity at slow temporal frequencies, and thus obscure evidence for temporal inhibition. I return to this issue in the discussion of Experiment 3. However, this issue does imply that the TCSF paradigm is perhaps less powerful than the two-pulse paradigm when used to determine evidence for temporal inhibition. Despite this, a finding of any evidence for temporal inhibition in the TCSF paradigm does rule out transienton-sustained inhibition as the sole mediator of temporal inhibition.

\section{Method}

Contrast sensitivities at seven temporal frequencies ranging from 2 to $24 \mathrm{~Hz}$ were obtained by flickering a digit around a gray background according to a sine wave weighted by a Gaussian envelope with a standard deviation of $83 \mathrm{msec}$. Example temporal functions are shown in Figure 6. The stimuli were presented on a Tektronix 604 oscilloscope with a fast $\mathrm{P} 15$ phosphor at a $4-\mathrm{msec}(250-\mathrm{Hz})$ refresh rate. To maintain a constant mean luminance, the formula for contrast was changed to contrast $=\left(\mathrm{L}_{\max }-\mathrm{L}_{\text {nin }}\right) /\left(\mathrm{L}_{\max }+\mathrm{L}_{\min }\right)$. 

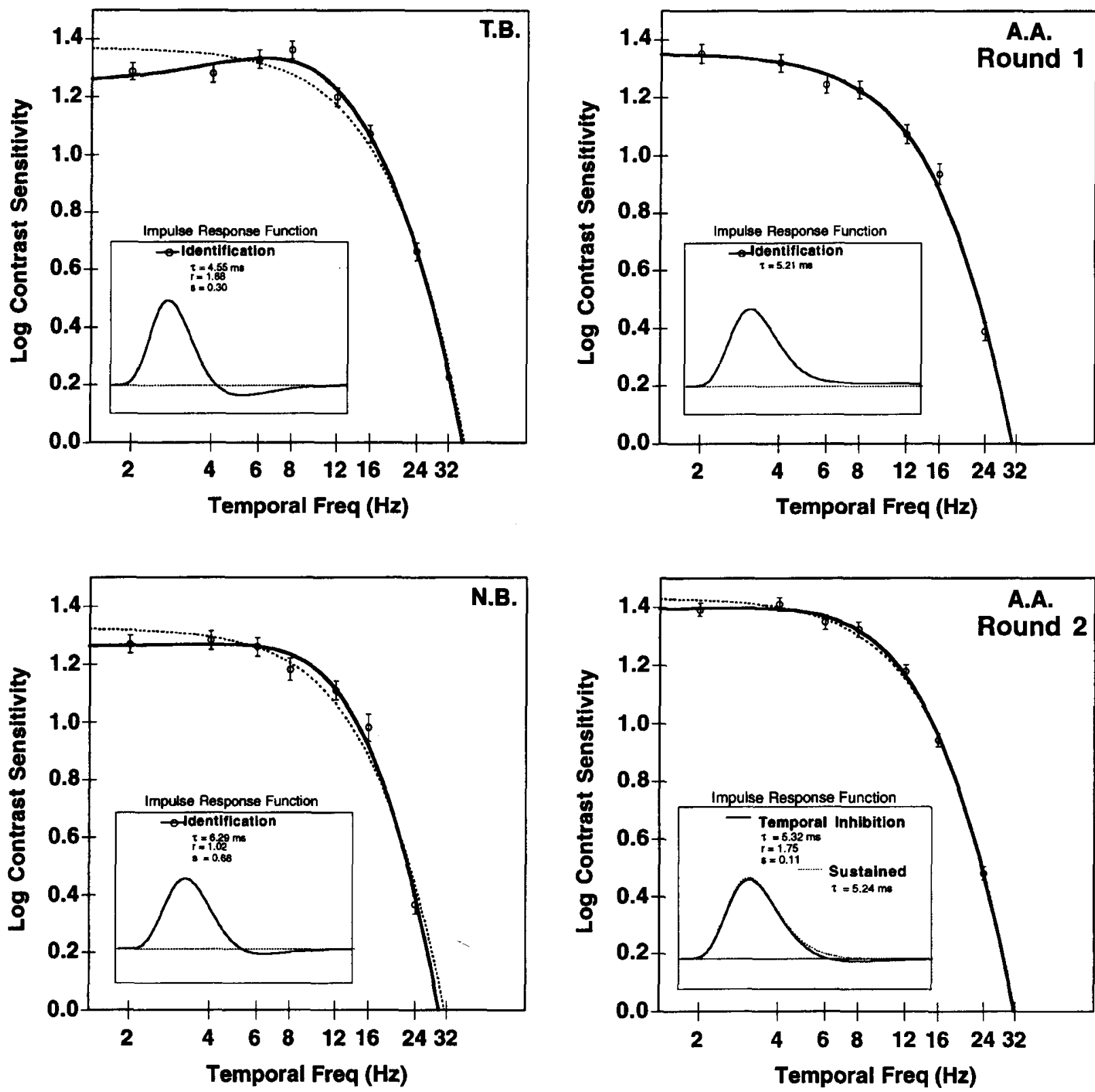

Figure 7. Temporal contrast sensitivity functions for 3 observers for Experiment 3 . The insets show the recovered impulse response functions. Error bars represent the standard deviation of the QUEST threshold estimate (Watson \& Pelli, 1983).

Observers. Three observers-the author (T.B.) and 2 graduate students (N.B. and A.A.) — completed 80 trials at each temporal frequency. A.A. was female.

Stimuli and Apparatus, Observers viewed an illuminated patch subtending $1.40^{\circ}$ vertically and $1.57^{\circ}$ horizontally on the face of a Tektronix 604 oscilloscope. The background luminance was fixed at $20 \mathrm{~cd} / \mathrm{m}^{2}$, about three times higher than in Experiments 1-2. Stimuli were a 2 and a 5 , rendered in the same Times Roman font used in Experiments 1-2. Observers viewed these stimuli from a distance of $86 \mathrm{~cm}$, which resulted in the digits subtending $0.50^{\circ}$ vertically and $0.37^{\circ}$ horizontally.

Design and Procedure. On each trial, the contrast of the pulses was determined by the same adaptive search technique (QUEST; Watson \& Pelli, 1983) as that used in Experiment 1, which deter- mines the stimulus contrast that affords $82 \%$ correct identification over trials. The resulting contrast threshold is converted to sensitivity by taking the logarithm of the inverse of the contrast threshold.

\section{Results and Discussion}

Figure 7 shows TCSF data from Experiment 3 for the 3 observers. Observer T.B.'s data showed a decrease in sensitivity at low temporal frequencies, which demonstrates clear evidence for temporal inhibition. Data from Observer N.B. demonstrated a flattening of the TCSF at low temporal frequencies; thus a model-based interpretation is necessary to determine whether a temporal in- 
hibition account is necessary to account for the data. Observer A.A. showed no sign of a fall-off at low temporal frequencies; therefore a temporal inhibition is not required to account for her data.

The Experiment 3 data were modeled by computing the Fourier transform of the impulse response function (Equation 2) and fitting a model that consists only of the impulse response function parameters $(\tau, r$, and $s$ ), along with a sensitivity parameter $g$ that scales the TCSF vertically. This is the transfer function $G(w)$ :

$$
G(w)=g\left|(1+2 i \pi \tau w)^{-n_{1}}-\frac{s}{(1+2 i \pi r \tau w)^{-n_{2}}}\right|,
$$

where $w$ is the temporal frequency flicker rate for a condition, $g$ is a sensitivity parameter and $\tau, r$, and $s$ are the impulse response function parameters from Equation 2 that determine the range of temporal frequencies that underlie a given task. The model fits are shown in Figure 7, along with the recovered impulse response functions. In some cases, the data did not require temporal inhibition, which was accomplished by setting $s$ to zero, eliminating the second part of Equation 7. Table 3 shows the bestfitting model parameters for each observer.

Observer T.B.'s data were not well fit by a monophasic impulse response function (dashed curve) and clearly demonstrate evidence for temporal inhibition. Observer N.B.'s data deviated from a monophasic prediction at the $2-\mathrm{Hz}$ flicker rate. One technique that can be used to determine whether temporal inhibition is required to account for Observer N.B.'s data is to compute confidence intervals around the impulse response function parameters. If the confidence intervals around the $s$ parameter do not include zero, temporal inhibition appears to be necessary to account for the character identification data.

Confidence intervals around the impulse response function parameters are determined as follows. A distribution of impulse response function parameters was created by using the variability estimates of the thresholds that come from the standard error of the mean for each threshold. From the threshold estimates and the standard error of the mean for each threshold, a new distribution of threshold estimates was constructed by selecting from a normal distribution with mean equal to each threshold and standard deviation equal to the standard error of the mean for that threshold. This created a new set of seven thresholds. The parameter fitting procedures were then applied to this new set of thresholds, and values of $\tau, r, s$, and $g$ were recorded. This procedure was repeated 200 times, and the resulting 200 values of the parameters construct a sampling distribution with some mean and standard deviation. These distributions can be used to construct confidence intervals around the parameter estimates for Observer N.B. The $95 \%$ confidence intervals for $\tau, r$, $s$, and $g$ were $6.29 \pm 0.397,1.02 \pm 0.0265,0.681 \pm$ 0.0471 , and $57.6 \pm 5.14$, respectively. Since the confidence interval for the $s$ parameter does not include zero, we conclude that Observer N.B.'s data require a model that includes temporal inhibition.

The data from Observer A.A. show no evidence of a fall-off in sensitivity at low temporal frequencies, and her data are well fit by a model that does not include temporal inhibition. Observer A.A. was not as well practiced as Observers N.B. and T.B. To verify that this difference did not influence her data, Observer A.A. replicated her results in a second round of data collection. The results are shown as Round 2 in Figure 7 and demonstrate that practice slightly improved her overall sensitivity, by an average of $0.075 \mathrm{log}$ units. However, the overall data pattern is the same. For a model that assumes temporal inhibition, the $95 \%$ confidence interval around the $s$ parameter includes $0(0.11 \pm 0.22)$. This indicates that the estimated value for the temporal inhibitory model component is not reliably different from zero and confirms the findings from Round 1: Observer A.A. did not show evidence of temporal inhibition in the character identification task. Observer A.A. had no evidence of any reading disorder, and her optical correction was quite small (a small, 1-diopter, myopia correction that corrected her to $20 / 20$ vision).

The Experiment 3 data demonstrate that, at least for some observers, temporal inhibition exists in tasks where there are no abrupt onsets. This suggests that the inhibitory mechanisms are not driven solely by abrupt onsets or offsets, but instead represent the intrinsic temporal properties of the pathways responsible for character identification. The evidence for temporal inhibition is not as strong as in Experiments 1 and 2, and perhaps better evidence for a fall-off at slow temporal frequencies would come from 1 - or even $.5-\mathrm{Hz}$ flicker rates. However, these stimuli were not used since they leave open the possibility that an eye movement will occur during the stimulus presentation. An eye movement has the tendency to introduce higher temporal frequencies into the stimulus as the eye moves across a fixed spatial pattern. These higher temporal frequencies are compatible with the temporal inhibitory responses and are therefore not reduced by the inhibitory portion, as are slower temporal frequencies. The result is an increase in sensitivity at very low temporal frequencies, diminishing the evidence for temporal inhibition. These mechanisms may have been at work in even the $500-\mathrm{msec}(2-\mathrm{Hz})$ presentations of Experiment 3, which may explain the relatively weak evidence for temporal inhibition in the TCSF data.

\section{GENERAL DISCUSSION}

Four general conclusions are possible from Experiments $1-3$. First, together these experiments provide clear evidence for temporal inhibitory processes in the mechanisms that subsume character detection and identification. The data in both the detection and identification tasks are consistent with the two-pulse detection literature findings, and the range of temporal inhibition 
delay intervals corresponds to the range reported in the literature (see Table 1). Thus it appears that digits are similar to disks and gratings in both detection and identification tasks.

Second, these results disconfirm a linear filter model with a monophasic impulse response function (Busey \& Loftus, 1994; Loftus et al., 1993) for three different tasks involving characters.

Third, when the previous character identification model was altered to allow for temporal inhibition, overall it provided a good account of the data in both experiments.

Fourth, the Experiment 3 data yielded evidence for temporal inhibition in stimuli that did not contain abrupt onsets, suggesting that the transient-on-sustained inhibition proposed by Breitmeyer and Ganz (1977) cannot be the sole mediator of temporal inhibition. In addition, transienton-sustained inhibition cannot explain the facilitatory processes, since the transient inhibition appears to affect both on- and off-center sustained neurons in the cat (Breitmeyer, 1984) and is thus indifferent to contrast polarity. An explanation based on interactions between the magnocellular and parvocellar parallel pathways that extend through the lateral geniculate nucleus cannot account for the temporal inhibition, since the temporal response properties of the neurons in each pathway are quite similar, and both demonstrate evidence for temporal inhibition (Hawken, Shapley, \& Grosof, 1996). The Experiment 3 data demonstrate that some form of withinchannel inhibition is sufficient to generate temporal inhibition. One possible mechanism is that proposed by Purcell and Stewart (1971), who suggested that lateral inhibitory mechanisms in the retina might underlie temporal inhibition. However, this does not rule out the contributions of cortical mechanisms to the temporal properties of the visual pathway responsible for character identification.

\section{Previous Linear Filter Modeling}

There are several reasons why a biphasic impulse response function might not have been necessary to successfully model previous character identification experiments. First, the background luminance might have been low enough that an inhibitory response was not generated. The background luminances of previous experiments (e.g., Busey \& Loftus, 1994; Loftus et al., 1993) were somewhat lower, $3.5 \mathrm{~cd} / \mathrm{m}^{2}$, which is below the approximate $5-\mathrm{cd} / \mathrm{m}^{2}$ background given by Sperling and Sondhi (1968) as necessary for the appearance of biphasic response functions. Second, the experiments used to test the linear filter model may not have been powerful enough to disconfirm a monophasic response function.

The version of the theory that includes a monophasic response function is a subset of all biphasic models; thus any data that are well fit by a monophasic version of the theory will also be fit by a biphasic version of the theory.

Despite the good fits of the linear filter version of the theory to the character identification data, the current con- ceptualization of the model is limited by the fact that there is no mechanism within the model to control parametrically the inhibitory component of the impulse response function with changes in the background luminance level. This is one advantage of the Sperling and Sondhi (1968) model. Perhaps the character identification model can be adapted to include their conceptualization of the impulse response model based on gain control mechanisms. As with most models of two-pulse performance, the amount of temporal inhibition is represented by a delayed inhibitory signal, although the physiological analogue of this signal remains to be characterized.

The results of these experiments demonstrate the need to consider the possibility of temporal inhibition in information processing tasks that are normally placed outside the domain of simple detection experiments. The present studies used near-threshold stimuli on relatively dim backgrounds and still revealed evidence for temporal inhibition. The detection literature demonstrates that as the background luminance increases, the amount of temporal inhibition increases as well. In addition, experiments by Bowen (1989) demonstrate that biphasic or even multiphasic impulse response functions are necessary to account for a phenomenon in which two suprathreshold pulses produce three apparent flashes. This suggests that inhibitory mechanisms are at work in luminance conditions normally associated with reading and other information processing tasks.

\section{Implications for Developmental Dyslexia and Transient/Sustained Inhibition}

The present work incorporates temporal inhibition into an information processing model, which is a first step toward linking the lower level visual and higher level cognitive mechanisms. Such links are particularly important for disorders such as developmental dyslexia, in which a deficit in high temporal frequencies has been found (Lovegrove et al., 1990; Williams \& Lecluyse, 1990). Previously it was unknown whether reading performance could be linked to the flicker-fusion experiments used to test temporal acuity and reading performance. Several authors (Breitmeyer, 1993; Lovegrove, 1993) have suggested that the detection and identification of letters and words is carried out by the parvocellular/ sustained system during eye fixations. The magnocellular/transient system is thought to inhibit the parvocellular/sustained system during each saccade, preventing the information from a previous fixation from overlapping with the new information from a new fixation. A deficit in the magnocellular system, as suggested by a loss in high temporal frequency sensitivity, suggests that this necessary inhibition is not taking place. However, Burr, Morrone, and Ross (1994) found evidence only for suppression of the magnocellular/transient system during eye movements, casting doubt on this theory. In addition, if the parvocellular/sustained system processes only 
higher spatial frequencies, these frequencies are effectively blurred during a rapid eye movement and need not be suppressed.

More recent studies have failed to show a transient deficit in adults and children with dyslexia. WaltherMüller (1995) tested dyslexic children on temporal contrast sensitivity, line-spread, visual persistence, and short-range motion tasks. He found no systematic differences between control and dyslexic groups in any of the four tasks. Hayduk, Bruck, and Cavanagh (1996) reported no differences between control and dyslexic children and adults on temporal contrast sensitivity and visual search tasks. Unfortunately, in both these studies the temporal contrast sensitivity measurements were conducted with a 2-cycle/degree grating. In two-pulse tasks, this spatial frequency shows evidence of temporal inhibition only at very high luminance levels $\left(150 \mathrm{~cd} / \mathrm{m}^{2}\right)$ (Ohtani \& Ejima, 1988). In Ohtani and Ejima's two-pulse experiment, no temporal inhibition was found for 1.5 cpd stimuli on background levels of $8 \mathrm{~cd} / \mathrm{m}^{2}$, which is close to the stimulus value of $14 \mathrm{~cd} / \mathrm{m}^{2}$ used by Hayduk et al. and the value of $11 \mathrm{~cd} / \mathrm{m}^{2}$ used by Walther-Muller. It is possible that the stimuli used to test dyslexic children did not produce temporal inhibition in either controls or dyslexics and therefore could not show a transience deficit. A grating with spatial frequencies larger than $.75 \mathrm{cpd}$ produced temporal inhibition at almost all adaptation levels in the Ohtani and Ejima study. The stimuli used in the search tasks of Hayduk et al. were $1^{\circ}$ flickering disks, and probably large enough to create temporal inhibition. However, this was a visual search task with suprathreshold stimuli and is therefore not directly comparable to the temporal contrast sensitivity task.

Despite the apparent inadequacy of the magnocellular/transient explanation of dyslexia, a link between a high temporal acuity loss and dyslexia might still be possible. Rayner and Pollatsek (1989), for example, have argued for a deficit in the eye movements of dyslexics, which may be driven by a mechanism that includes temporal inhibition. The present experiments demonstrate that information processing tasks rely on temporal inhibition, which is a mechanism that improves temporal acuity. If temporal inhibition plays a central role in reading mechanisms, this would account for the observed correlation between dyslexia and a loss in temporal acuity. Temporal inhibition may inhibit processing in a single pathway and prevent information from one eye fixation from persisting into the next. One possible test of this hypothesis is to determine whether persons with dyslexia produce evidence for temporal inhibition in a two-pulse or TCSF experiment. The entire temporal contrast sensitivity function should be mapped out for both control and dyslexic subjects using a .75-cpd grating, and the control subjects should show a bandpass TCSF, whereas the dyslexic subjects should show a low-pass TCSF if a transience deficit is to be demonstrated. The results from Observer A.A. in Experiment 3 suggest that such a link may not exist: She showed no evidence of transience in her TCSF, yet she does not suffer from dyslexia. Clearly, more evidence from a wider range of control and dyslexic observers is necessary to fully answer this question.

\section{REFERENCES}

Alexander, K., XIE, W., \& Derlacki, D. (1994). Spatial frequency characteristics of letter identification. Journal of the Optical Society of America A, 11, 2375-2382.

Bergen, J. R., \& Wilson, H. R. (1985). Prediction of flicker sensitivities from temporal three-pulse data. Vision Research, 25, 577-582.

BlaCKWELL, H. R. (1963). Neural theories of simple visual discriminations. Journal of the Optical Society of America, 53, 129-160.

Bouman, M. A., \& VAN DEN BRINK, G. (1952). On the integrate capacity in time and space of the human peripheral retina. Journal of the Optical Society of America, 42, 617-620.

Bowen, R. W. (1989). Two pulses seen as three flashes: A superposition analysis. Vision Research, 29, 409-417.

BOYNTON, R. M. (1972). Discrimination of homogeneous double pulses of light. In L. M. Hurvich \& D. Jameson (Eds.), Handbook of sensory physiology (pp. 202-232). New York: Springer-Verlag.

BREITMEYER, B. G. (1984). Visual masking: An integrative approach New York: Oxford University Press.

BREITMEYER, B. G. (1993). The roles of sustained (P) and transient (M) channels in reading and reading disability. In S. F. Wright \& R. Groner (Ed.), Facets of dyslexia and its remediation (pp. 13-31). Amsterdam: Elsevier.

BreitMeyer, B. G., \& Ganz, L. (1977). Temporal studies with flashed gratings: Inferences about human transient and sustained channels. Vision Research, 17, 861-865.

BurR, D. C., \& Morrone, M. C. (1993). Impulse-response functions for chromatic and achromatic stimuli. Journal of the Optical Society of America A, 10, 1706-1713.

Burr, D. C., Morrone, M. C., \& Ross, J. (1994). Selective suppression of the magnocellular visual pathway during saccadic eye movements. Nature, 371, 511-513.

BuSEY, T. A., \& LofTus, G. R. (1994). Sensory and cognitive components of visual information acquisition. Psychological Review, 101, 446-469.

Coltheart, M. (1980). Iconic memory and visible persistence. Perception \& Psychophysics, 27, 183-228.

DEN BRINKER, A.C. (1989). A comparison of results from parameter estimations of impulse responses of the transient visual system. Biological Cybernetics, 61, 139-151.

DEN BRINKER, A.C. (1990). Changes with background in the linear model of the transient visual system. Biological Cybernetics, 62, 441-451.

Enroth-Cugell, C., Robson, J. G., Schweitzer-Tong, D. E., \& WATson, A. B. (1983). Spatiotemporal interactions in cat retinal ganglion cells showing linear spatial summation. Journal of Physiology. 341, 279-301.

Eriksen, C. W., \& Schultz, D. W. (1979). Information processing in visual search: A continuous flow conception and experimental results. Perception \& PSychophysics, 25, 249-263.

FISHER, D. (1982). Limited-channel models of automatic detection. Capacity and scanning in visual search. Psychological Review, 89, 662692.

Gegenfurtner, K., \& Sperling, G. (1993). Information transfer in iconic memory experiments. Journal of Experimental Psychology: Human Perception \& Performance, 19, 845-866.

GEORGESon, M. A. (1987). Temporal properties of spatial contrast vision. Vision Research, 27, 765-780.

Hawken, M. J., Shapley, R. M., \& Grosof, D. H. (1996). Temporalfrequency selectivity in monkey visual cortex. Visual Neuroscience. 13, 477-492. 
Hayduk, S., Bruck, M., \& Cavanagh, P. (1996). Low-level visual processing skills of adults and children with dyslexia. Cognitive Neuropsychology, 13, 975-1015.

Hogben, J. H., \& Di Lollo, V. (1974). Perceptual integration and perceptual segregation of brief visual stimuli. Vision Research, 14, 1059-1069.

IKEDA, M. (1965). Temporal summation of positive and negative flashes in the visual system. Journal of the Optical Society of America, 55, $1527-1534$.

IKEDA, M. (1986). Temporal impulse response. Vision Research, 26, $1431-1440$.

KraUSKOPF, J. (1980). Discrimination and detection of changes in luminance. Vision Research, 20, 671-677.

Loftus, G. R., Busey, T. A., \& Senders, J. W. (1993). Providing a sensory basis for models of visual information acquisition. Perception \& Psychophysics, 54, 535-554.

LofTUs, G. R., \& IRWIN, D. E. (1998). On the relations among different measures of visible and informational persistence. Cognitive Psychology, 35, 135-199.

Loftus, G. R., \& RuthrufF, E. (1994). A theory of visual information acquisition and visual memory with special application to intensityduration tradeoffs. Journal of Experimental Psychology: Human Perception \& Performance, 20, 33-49.

Lovegrove, W. (1993). Do dyslexics have a visual deficit? In S. F. Wright \& R. Groner (Ed.), Facets of dyslexia and its remediation (pp. 33-49). Amsterdam: Elsevier.

Lovegrove, W., Garzia, R., \& Nicholson, S. (1990). Experimental evidence for a transient system deficit in specific reading disability. Journal of the American Optometric Association, 16, 137-146.

MATIN, L., \& BowEN, R. W. (1976). Measuring the duration of perception. Perception \& Psychophysics, 20, 66-76.

MEIJER, J. G., vaN DER Wildt, G., \& VAN DEN Brink, G. (1978). Twinflash response as a function of flash diameter. Vision Research, 18, 1111-1116.

MusselWhite, M. J., \& JefFREYS, D. A. (1983). Visual evoked potentials to double-pulse pattern presentation. Vision Research, 23, 135-143.

OHTANi, Y., \& EıIMA, Y. (1988). Relation between flicker and two-pulse sensitivities for sinusoidal gratings. Vision Research, 28, 145-156.

PELLI, D. G. (1997). The VideoToolbox software for visual psychophysics: Transforming numbers into movies. Spatial Vision, 10, 437-442.

PELLI, D. G., \& ZhANG, L. (1991). Accurate control of contrast on microcomputer displays. Vision Research, 30, 1033-1048.

Purcell, D. G., \& StewarT, A. L. (1971). The two-flash threshold: An evaluation of critical-duration and visual-persistence hypotheses. Perception \& Psychophysics, 9, 61-64.

RASHBASS, C. (1970). The visibility of transient changes of luminance. Journal of Physiology, 210, 165-186.

Rayner, K., \& Pollatsek, A. (1989). The psychology of reading. Englewood Cliffs, NJ: Prentice-Hall.

RouFs, J. A. J. (1973). Dynamic properties of vision-III. Twin flashes, single flashes and flicker fusion. Vision Research, 13, 309-323.

Roufs, J. A. J., \& BlommaerT, F. J. J. (1981). Temporal impulse and step responses of the human eye obtained psychophysically by means of a drift-correcting perturbation technique. Vision Research, 21, 1203-1221.
RumelhaRT, D. E. (1970). A multicomponent theory of the perception of briefly exposed visual displays. Journal of Mathematical Psychology, 7, 191-218.

SMIrH, P. L. (1995). Psychophysically principled models of visual simple reaction time. Psychological Review, 102, 567-593.

Solomon, J. A., \& Pelli, D. G. (1994). The visual filter mediating letter identification. Nature, 369, 395-397.

SPERLING, G. (1960). The information available in brief visual presentations. Psychological Monographs, 74, 1-29.

SPERLING, G., \& SoNDHI, M. M. (1968). Model for visual luminance discrimination and flicker detection. Journal of the Optical Society of America, 58, 1133-1145.

TheODOR, L. H. (1972). The detectability of a brief gap in a pulse of light as a function of its temporal location within the pulse. Perception \& Psychophysics, 12, 168-170.

ToWNSEND, J. T. (1981). Some characteristics of visual whole report behavior. Acta Psychologica, 47, 149-173.

UCHIKAWA, K., \& IKEDA, M. (1986). Temporal integration of chromatic double pulses for detection of equal-luminance wavelength changes. Journal of the Optical Society of America A, 3, 2109-2115.

WALTHER-MÜLLER, P. U. (1995). Is there a deficit of early vision in dyslexia? Perception, 24, 919-936.

Watson, A. B. (1978). Probability summation over time. Vision Research, 19, 515-522.

WATsON, A. B. (1986). Temporal sensitivity. In K. R. Boff, L. Kaufman, \& J. P. Thomas (Eds.), Handbook of perception and human performance (Vol. 1, pp. 6.1-6.42). New York: Wiley.

Watson, A. B., \& NaChmias, J. (1977). Patterns of temporal interaction in the detection of gratings. Vision Research, 17, 893-902.

WATson, A. B., \& Pelli, D. G. (1983). QUEST: A Bayesian adaptive psychometric method. Perception \& Psychophysics, 33, 113-120.

Williams, M., \& Lecluyse, K. (1990). Perceptual consequences of a temporal processing deficit in reading disabled children. Journal of the American Optometric Association, 61, 111-121.

\section{NOTES}

1. At threshold, observers often cannot determine the sign of the stimulus. Krauskopf (1980) and Rashbass (1970) demonstrated this effect. In addition, Alexander Cogan (Cogan, personal communication, October 13, 1997) has reported that above threshold when observers are $100 \%$ correct regarding which of two intervals contained the stronger brightness change, they are up to $40 \%$ in error as to the sign (i.e., whether the increment or the decrement made the change stronger).

2. An ISI of zero for the same-contrast condition amounts to a single pulse, which is readily modeled like any other stimulus condition. An ISI of zero for the different-contrast condition was simply a positivecontrast pulse for $30 \mathrm{msec}$ followed immediately by a negative-contrast pulse (or a negative- followed by a positive-contrast pulse).

3 . The guessing formula is $p=(x-.1) / .9$, where $x$ is the raw proportion correct and $p$ is the corrected proportion.

(Manuscript received July 3, 1997; revision accepted for publication November 3,1997 .) 